\title{
Optimal error estimate for a space-time discretization for incompressible generalized Newtonian fluids: the Dirichlet problem
}

\author{
Luigi C. Berselli ${ }^{1} \cdot$ Michael Růžička ${ }^{2}$ \\ Published online: 9 August 2021 \\ (C) The Author(s) 2021
}

\begin{abstract}
In this paper we prove optimal error estimates for solutions with natural regularity of the equations describing the unsteady motion of incompressible shear-thinning fluids. We consider a full space-time semi-implicit scheme for the discretization. The main novelty, with respect to previous results, is that we obtain the estimates directly without introducing intermediate semi-discrete problems, which enables the treatment of homogeneous Dirichlet boundary conditions.
\end{abstract}

Keywords Space-time discretization · Generalized Newtonian fluids · Error analysis

Mathematics Subject Classification 65M60 $\cdot 65 \mathrm{M} 15 \cdot 35 \mathrm{~B} 35$

\section{Introduction}

In this paper we study a space-time discretization of the unsteady system describing the motion of homogeneous (for simplicity the density $\rho$ is set equal to 1), incompressible shear-thinning fluids under homogeneous Dirichlet boundary conditions. We prove optimal error estimates (cf. Sect. 2.4) for solutions possessing a natural regularity, extending the results in [5] to the case of homogeneous Dirichlet boundary conditions. Our method differs from most previous investigations in as much as we use no intermediate semidiscrete problems to prove our result. We restrict ourselves to the three-dimensional setting, however, all results can be easily adapted to the general setting in $d$-dimensions.

This article is part of the topical collection dedicated to In Honor of Professor Hideo Kozono's 60th Birthday edited by Kazuhiro Ishige, Tohru Ozawa, Senjo Shimizu, and Yasushi Taniuchi.

Michael Růžička

rose@mathematik.uni-freiburg.de

Luigi C. Berselli

luigi.carlo.berselli@unipi.it

1 Dipartimento di Matematica, Università di Pisa, Via F. Buonarroti 1/c, 56127 Pisa, Italy

2 Institute of Applied Mathematics, Albert-Ludwigs-University Freiburg, Ernst-Zermelo-Str. 1, 79104 Freiburg, Germany 
More precisely, we consider for a bounded polyhedral domain $\Omega \subset \mathbb{R}^{3}$ and a finite time interval $I:=(0, T)$, for some given $T>0$, the system

$$
\begin{aligned}
\partial_{t} \mathbf{u}-\operatorname{div} \mathbf{S}(\mathbf{D u})+[\nabla \mathbf{u}] \mathbf{u}+\nabla q & =\mathbf{f} & & \text { in } I \times \Omega, \\
\operatorname{div} \mathbf{u} & =0 & & \text { in } I \times \Omega, \\
\mathbf{u}(0) & =\mathbf{u}_{0} & & \text { in } \Omega,
\end{aligned}
$$

where the vector field $\mathbf{u}=\left(u_{1}, u_{2}, u_{3}\right)^{\top}$ is the velocity, the scalar $q$ is the kinematic pressure, the vector $\mathbf{f}=\left(f_{1}, f_{2}, f_{3}\right)^{\top}$ is the external body force and $\mathbf{u}_{0}$ is the initial velocity. We assume that the extra stress tensor $\mathbf{S}$ has $(p, \delta)$-structure for some $p \in(1,2]$, and $\delta \in[0, \infty)$ (cf. Sect. 2.2). For the convective term we use the notation $([\nabla \mathbf{u}] \mathbf{u})_{i}=\sum_{j=1}^{3} u_{j} \partial_{j} u_{i}$, $i=1,2,3$, while $\mathbf{D u}:=\frac{1}{2}\left(\nabla \mathbf{u}+\nabla \mathbf{u}^{\top}\right)$ denotes the symmetric part of the gradient $\nabla \mathbf{u}$. For smooth enough solutions $(\mathbf{u}, q)$ the variational formulation of (1.1) reads

$$
\begin{aligned}
\left(\partial_{t} \mathbf{u}(t), \mathbf{v}\right)+(\mathbf{S}(\mathbf{D u}(t)), \mathbf{D} \mathbf{v})+b(\mathbf{u}(t), \mathbf{u}(t), \mathbf{v})-(q(t), \operatorname{div} \mathbf{v}) & =(\mathbf{f}(t), \mathbf{v}), \\
(\operatorname{div} \mathbf{u}(t), \eta) & =0 \\
(\mathbf{u}(0), \mathbf{v}) & =\left(\mathbf{u}_{0}, \mathbf{v}\right)
\end{aligned}
$$

for all $\mathbf{v} \in V:=W_{0}^{1, p}(\Omega)^{3}, \eta \in Q:=L_{0}^{p^{\prime}}(\Omega)$ and almost every $t \in I$. We used the notation

$$
b(\mathbf{u}, \mathbf{v}, \mathbf{w}):=\frac{1}{2}[([\nabla \mathbf{v}] \mathbf{u}, \mathbf{w})-([\nabla \mathbf{w}] \mathbf{u}, \mathbf{v})],
$$

for the convective term to have a stable space-discretization. Note that $b(\cdot, \cdot, \cdot)$ is skewsymmetric with respect to the last two arguments, i.e., $b(\mathbf{u}, \mathbf{v}, \mathbf{w})=-b(\mathbf{u}, \mathbf{w}, \mathbf{v})$ and that for solenoidal functions it holds $b(\mathbf{u}, \mathbf{v}, \mathbf{w})=([\nabla \mathbf{v}] \mathbf{u}, \mathbf{w})$. We perform an error analysis for the semi-implicit space-time discretization, which for given $h>0$ and $M \in \mathbb{N}$ reads: for $\mathbf{u}_{h}^{0}:=\Pi_{h}^{\text {div }} \mathbf{u}_{0}$ find $\left(\mathbf{u}_{h}^{m}, q_{h}^{m}\right) \in V_{h} \times Q_{h}, m=1, \ldots, M$, such that for all $\mathbf{v}_{h} \in V_{h}, \eta_{h} \in Q_{h}$ holds

$$
\begin{aligned}
\left(d_{t} \mathbf{u}_{h}^{m}, \mathbf{v}_{h}\right)+\left(\mathbf{S}\left(\mathbf{D} \mathbf{u}_{h}^{m}\right), \mathbf{D} \mathbf{v}_{h}\right)+b\left(\mathbf{u}_{h}^{m-1}, \mathbf{u}_{h}^{m}, \mathbf{v}_{h}\right)-\left(q_{h}^{m}, \operatorname{div} \mathbf{v}_{h}\right) & =\left(\mathbf{f}\left(t_{m}\right), \mathbf{v}_{h}\right), \\
\left(\operatorname{div} \mathbf{u}_{h}^{m}, \eta_{h}\right) & =0,
\end{aligned}
$$

where $d_{t} \mathbf{u}_{h}^{m}:=\kappa^{-1}\left(\mathbf{u}_{h}^{m}-\mathbf{u}_{h}^{m-1}\right)$ is the backward difference quotient with $\kappa:=\frac{T}{M}$. Here $V_{h} \subset V, Q_{h} \subset Q$ are appropriate stable finite element spaces with mesh size $h>0$. The precise setup can be found in Sect. 2.3.

\section{Preliminaries and main results}

In this section we introduce the notation, the setup, and recall some technical results which will be needed in the proof of the main result.

\subsection{Function spaces}

We use $c, C$ to denote generic constants, which may change from line to line, but are not depending on the crucial quantities. We write $f \sim g$ if and only if there exist constants $c, C>0$ such that $c f \leq g \leq C f$.

We will use the customary Lebesgue spaces $\left(L^{p}(\Omega),\|\cdot\|_{p}\right)$ and Sobolev spaces $\left(W^{k, p}(\Omega),\|\cdot\|_{k, p}\right), k \in \mathbb{N}$. We do not distinguish between scalar, vector-valued or tensor-valued 
function spaces in the notation if there is no danger of confusion. However, we denote vectorvalued functions by small boldfaced letters and tensor-valued functions by capital boldfaced letters. If a norm is considered on a set $M$ different than $\Omega$ we indicate this in the respective norms as $\|\cdot\|_{p, M},\|\cdot\|_{k, p, M}$. We equip $W_{0}^{1, p}(\Omega)$ with the gradient norm $\|\nabla \cdot\|_{p}$. We denote by $|M|$ the 3dimensional Lebesgue measure of a measurable set $M$. The mean value of a locally integrable function $f$ over a measurable set $M \subset \Omega$ is denoted by $\langle f\rangle_{M}:=f_{M} f d x=\frac{1}{|M|} \int_{M} f d x$. By $L_{0}^{p}(\Omega)$ we denote the space of functions $f \in L^{p}(\Omega)$ with $\langle f\rangle_{\Omega}=0$. Moreover, we use the notation $(f, g):=\int_{\Omega} f g d x$, whenever the right-hand side is well-defined.

We use the following notation

$$
X:=\left(W^{1, p}(\Omega)\right)^{3}, \quad V:=\left(W_{0}^{1, p}(\Omega)\right)^{3}, \quad V_{\mathrm{div}}:=\{\mathbf{v} \in V \mid \operatorname{div} \mathbf{v}=0\}, \quad Y:=L^{p^{\prime}}(\Omega), \quad Q:=L_{0}^{p^{\prime}}(\Omega),
$$

for the most often used function spaces.

\subsection{Basic properties of the extra stress tensor}

For a tensor $\mathbf{P} \in \mathbb{R}^{3 \times 3}$ we denote its symmetric part by $\mathbf{P}^{\text {sym }}:=\frac{1}{2}\left(\mathbf{P}+\mathbf{P}^{\top}\right) \in \mathbb{R}_{\text {sym }}^{3 \times 3}:=\left\{\mathbf{A} \in \mathbb{R}^{3 \times 3} \mid \mathbf{P}=\mathbf{P}^{\top}\right\}$. The scalar product between two tensors $\mathbf{P}, \mathbf{Q}$ is denoted by $\mathbf{P} \cdot \mathbf{Q}$, and we use the notation $|\mathbf{P}|^{2}=\mathbf{P} \cdot \mathbf{P}$. We assume that the extra stress tensor $\mathbf{S}$ has $(p, \delta)$-structure, which will be defined now. A detailed discussion and full proofs of the following results can be found in $[12,21]$.

Assumption 2.1 We assume that $\mathbf{S}: \mathbb{R}^{3 \times 3} \rightarrow \mathbb{R}_{\mathrm{sym}}^{3 \times 3}$ belongs to $C^{0}\left(\mathbb{R}^{3 \times 3}\right.$; $\left.\mathbb{R}_{\text {sym }}^{3 \times 3}\right) \cap C^{1}\left(\mathbb{R}^{3 \times 3} \backslash\{\mathbf{0}\} ; \mathbb{R}_{\text {sym }}^{3 \times 3}\right)$, satisfies $\mathbf{S}(\mathbf{P})=\mathbf{S}\left(\mathbf{P}^{\text {sym }}\right)$ and $\mathbf{S}(\mathbf{0})=\mathbf{0}$. Moreover, we assume that $\mathbf{S}$ has $(p, \delta)$-structure, i.e., there exist $p \in(1, \infty), \delta \in[0, \infty)$, and constants $C_{0}, C_{1}>0$ such that

$$
\begin{gathered}
\sum_{i, j, k, l=1}^{3} \partial_{k l} S_{i j}(\mathbf{P}) Q_{i j} Q_{k l} \geq C_{0}\left(\delta+\left|\mathbf{P}^{\mathrm{sym}}\right|\right)^{p-2}\left|\mathbf{Q}^{\mathrm{sym}}\right|^{2}, \\
\left|\partial_{k l} S_{i j}(\mathbf{P})\right| \leq C_{1}\left(\delta+\left|\mathbf{P}^{\mathrm{sym}}\right|\right)^{p-2}
\end{gathered}
$$

are satisfied for all $\mathbf{P}, \mathbf{Q} \in \mathbb{R}^{3 \times 3}$ with $\mathbf{P}^{\text {sym }} \neq \mathbf{0}$ and all $i, j, k, l=1, \ldots, 3$. The constants $C_{0}$, $C_{1}$, and $p$ are called the characteristics of $\mathbf{S}$.

Remark 2.2 We would like to emphasize that, if not otherwise stated, the constants in the paper depend only on the characteristics of $\mathbf{S}$, but are independent of $\delta \geq 0$.

Another important tool are shifted $\mathrm{N}$-functions ${ }^{1}\left\{\varphi_{a}\right\}_{a \geq 0}$, cf. [12, 13, 21]. Defining for $t \geq 0$ a special $\mathrm{N}$-function $\varphi$ by

$$
\varphi(t):=\int_{0}^{t} \varphi^{\prime}(s) d s \quad \text { with } \quad \varphi^{\prime}(t):=(\delta+t)^{p-2} t,
$$

\footnotetext{
${ }^{1}$ A function $\psi: \mathbb{R}^{\geq 0} \rightarrow \mathbb{R}^{\geq 0}$ is called $N$-function if it is a continuous, convex function such that $\lim _{t \rightarrow 0} \frac{\psi(t)}{t}=0, \lim _{t \rightarrow \infty} \frac{\psi(t)}{t}=\infty$, and $\psi(t)>0$ for $t>0$.
} 
we can replace $C_{i}\left(\delta+\left|\mathbf{P}^{\text {sym }}\right|\right)^{p-2}$ in the right-hand side of (2.1) by $\widetilde{C}_{i} \varphi^{\prime \prime}\left(\left|\mathbf{P}^{\text {sym }}\right|\right)$, with some constants $\widetilde{C}_{i}>0, i=0,1$. Next, the shifted N-functions are defined for $t \geq 0$ by

$$
\varphi_{a}(t):=\int_{0}^{t} \varphi_{a}^{\prime}(s) d s \quad \text { with } \quad \varphi_{a}^{\prime}(t):=\varphi^{\prime}(a+t) \frac{t}{a+t} .
$$

Note that $\varphi_{a}(t) \sim(\delta+a+t)^{p-2} t^{2}$ and that the complementary function satisfies $\left(\varphi_{a}\right)^{*}(t) \sim\left((\delta+a)^{p-1}+t\right)^{p^{\prime}-2} t^{2}$. Moreover, the N-functions $\varphi_{a}$ and $\left(\varphi_{a}\right)^{*}$ satisfy the $\Delta_{2}-$ condition $^{2}$ uniformly with respect to $a \geq 0$, i.e., $\Delta_{2}\left(\varphi_{a}\right) \leq c 2^{\max \{2, p\}}$ and $\Delta_{2}\left(\left(\varphi_{a}\right)^{*}\right) \leq c 2^{\max \left\{2, p^{\prime}\right\}}$, respectively. We will use also Young's inequality: for all $\varepsilon>0$ there exists $c_{\epsilon}>0$, such that for all $s, t, a \geq 0$ it holds

$$
\begin{aligned}
t s & \leq \epsilon \varphi_{a}(t)+c_{\epsilon}\left(\varphi_{a}\right)^{*}(s), \\
t \varphi_{a}^{\prime}(s)+\varphi_{a}^{\prime}(t) s & \leq \epsilon \varphi_{a}(t)+c_{\epsilon} \varphi_{a}(s) .
\end{aligned}
$$

Closely related to the extra stress tensor $\mathbf{S}$ with $(p, \delta)$-structure is the function $\mathbf{F}: \mathbb{R}^{3 \times 3} \rightarrow$ $\mathbb{R}_{\text {sym }}^{3 \times 3}$ defined through

$$
\mathbf{F}(\mathbf{P}):=\left(\delta+\left|\mathbf{P}^{\mathrm{sym}}\right|\right)^{\frac{p-2}{2}} \mathbf{P}^{\mathrm{sym}} .
$$

In the following lemma we recall several useful results, which will be frequently used in the paper. The proofs of these results and more details can be found in [2, 12, 13, 21].

Proposition 2.3 Let $\mathbf{S}$ satisfy Assumption 2.1, let $\varphi$ be defined in (2.2), and let $\mathbf{F}$ be defined in (2.5).

(i) For all $\mathbf{P}, \mathbf{Q} \in \mathbb{R}^{3 \times 3}$

$$
\begin{aligned}
(\mathbf{S}(\mathbf{P})-\mathbf{S}(\mathbf{Q})) \cdot(\mathbf{P}-\mathbf{Q}) & \sim|\mathbf{F}(\mathbf{P})-\mathbf{F}(\mathbf{Q})|^{2}, \\
& \sim \varphi_{\left|\mathbf{P}^{\mathrm{sym}}\right|}\left(\left|\mathbf{P}^{\mathrm{sym}}-\mathbf{Q}^{\mathrm{sym}}\right|\right), \\
& \sim \varphi^{\prime \prime}\left(\left|\mathbf{P}^{\mathrm{sym}}\right|+\left|\mathbf{Q}^{\mathrm{sym}}\right|\right)\left|\mathbf{P}^{\mathrm{sym}}-\mathbf{Q}^{\mathrm{sym}}\right|^{2}, \\
\mathbf{S}(\mathbf{Q}) \cdot \mathbf{Q} & \sim|\mathbf{F}(\mathbf{Q})|^{2} \sim \varphi\left(\left|\mathbf{Q}^{\mathrm{sym}}\right|\right), \\
|\mathbf{S}(\mathbf{P})-\mathbf{S}(\mathbf{Q})| & \sim \varphi_{\left|\mathbf{P}^{\mathrm{sym}}\right|}^{\prime}\left(\left|\mathbf{P}^{\mathrm{sym}}-\mathbf{Q}^{\mathrm{sym}}\right|\right) .
\end{aligned}
$$

The constants depend only on the characteristics of $\mathbf{S}$.

(ii) For all $\epsilon>0$, there exist a constant $c_{\epsilon}>0$ (depending only on $\epsilon>0$ and on the characteristics of $\mathbf{S}$ ) such that for all $\mathbf{u}, \mathbf{v}, \mathbf{w} \in X$ we have

$$
\begin{aligned}
& (\mathbf{S}(\mathbf{D u})-\mathbf{S}(\mathbf{D v}), \mathbf{D w}-\mathbf{D v}) \leq \epsilon\|\mathbf{F}(\mathbf{D u})-\mathbf{F}(\mathbf{D v})\|_{2}^{2}+c_{\epsilon}\|\mathbf{F}(\mathbf{D w})-\mathbf{F}(\mathbf{D v})\|_{2}^{2}, \\
& (\mathbf{S}(\mathbf{D u})-\mathbf{S}(\mathbf{D v}), \mathbf{D w}-\mathbf{D v}) \leq \epsilon\|\mathbf{F}(\mathbf{D w})-\mathbf{F}(\mathbf{D v})\|_{2}^{2}+c_{\epsilon}\|\mathbf{F}(\mathbf{D u})-\mathbf{F}(\mathbf{D v})\|_{2}^{2},
\end{aligned}
$$

and for all $\mathbf{P}, \mathbf{Q} \in \mathbb{R}_{\mathrm{sym}}^{3 \times 3}, t \geq 0$ it holds

\footnotetext{
${ }^{2}$ An $\mathrm{N}$-function $\psi$ satisfies the $\Delta_{2}$-condition if there exists a constant $K$ such that $\psi(2 t) \leq K \psi(t)$ for all $t \geq 0$. The smallest such constant is denoted by $\Delta_{2}(\psi)$.
} 


$$
\begin{gathered}
\varphi_{|\mathbf{Q}|}(t) \leq c_{\varepsilon} \varphi_{|\mathbf{P}|}(t)+\varepsilon|\mathbf{F}(\mathbf{Q})-\mathbf{F}(\mathbf{P})|^{2}, \\
\left(\varphi_{|\mathbf{Q}|}\right)^{*}(t) \leq c_{\varepsilon}\left(\varphi_{|\mathbf{P}|}\right)^{*}(t)+\varepsilon|\mathbf{F}(\mathbf{Q})-\mathbf{F}(\mathbf{P})|^{2} .
\end{gathered}
$$

(iii) For all $\mathbf{H} \in L^{p}(\Omega)$ there holds

$$
\int_{\Omega}\left|\mathbf{F}(\mathbf{H})-\langle\mathbf{F}(\mathbf{H})\rangle_{\Omega}\right|^{2} d x \sim \int_{\Omega}\left|\mathbf{F}(\mathbf{H})-\mathbf{F}\left(\langle\mathbf{H}\rangle_{\Omega}\right)\right|^{2} d x,
$$

with constants depending only on p.

Let us recall the following result, taken from [16, Lemma 8], [4, Lemma 4.1], which is valid for $p \leq 2$.

Lemma 2.4 Let $\mathbf{S}$ satisfy Assumption 2.1 with $p \in(1,2]$ and $\delta \in[0, \infty)$. Then, there exists a constant c, depending only on the characteristics of $\mathbf{S}$, such that for sufficiently smooth $\mathbf{u}$, $\mathbf{v}$ there holds

$$
\|\mathbf{F}(\mathbf{D u})-\mathbf{F}(\mathbf{D v})\|_{2}^{2} \geq c\left(\delta+\|\mathbf{D u}\|_{p}+\|\mathbf{D u}-\mathbf{D v}\|_{p}\right)^{p-2}\|\mathbf{D u}-\mathbf{D} \mathbf{v}\|_{p}^{2} .
$$

\subsection{Discretizations}

For the time-discretization, given $T>0$ and $M \in \mathbb{N}$, we define the time step size as $\kappa:=T / M>0$, with the corresponding net $I^{M}:=\left\{t_{m}\right\}_{m=0}^{M}, t_{m}:=m \kappa$. We use the notation $I_{m}:=\left(t_{m-1}, t_{m}\right]$, with $m=1, \ldots, M$. For a given sequence $\left\{\mathbf{v}^{m}\right\}_{m=0}^{M}$ we define the backward differences quotient as

$$
d_{t} \mathbf{v}^{m}:=\frac{\mathbf{v}^{m}-\mathbf{v}^{m-1}}{\kappa}, \quad m=1, \ldots, M
$$

The proof of the main result uses the following modification of Gronwall's lemma.

Lemma 2.5 Let $1<p \leq 2$ and $T \in(0, \infty)$. For $M \in \mathbb{N}$ and $h>0$ let be given non-negative sequences $\left\{a_{m}(h)\right\}_{m=0}^{M},\left\{b_{m}(h)\right\}_{m=0}^{M},\left\{r_{m}(h, \kappa)\right\}_{m=1}^{M},\left\{s_{m}(h, \kappa)\right\}_{m=1}^{M},\left\{\rho_{m}(h, \kappa)\right\}_{m=1}^{M}$ and $\left\{\sigma_{m}(h, \kappa)\right\}_{m=1}^{M}$, where $\kappa:=\frac{T}{M}$. Assume that there exists $\mu_{0}, \widehat{\kappa}>0$ such that for all $0<h<1 / \sqrt{\mu_{0}}$ and all $0<\kappa<\widehat{\kappa}$ there holds:

$$
\begin{aligned}
\left(a_{0}(h)\right)^{2} & \leq \mu_{0} h^{2}, & \left(b_{0}(h)\right)^{2} & \leq \mu_{0} h^{2}, \\
\kappa \sum_{m=1}^{M}\left(r_{m}(h, \kappa)\right)^{2} & \leq \mu_{0} h^{2}, & \kappa \sum_{m=1}^{M}\left(s_{m}(h, \kappa)\right)^{2} & \leq \mu_{0} h^{2}, \\
\kappa \sum_{m=1}^{M}\left(\rho_{m}(h, \kappa)\right)^{2} & \leq \mu_{0} \kappa^{2}, & \kappa \sum_{m=1}^{M}\left(\sigma_{m}(h, \kappa)\right)^{2} & \leq \mu_{0} \kappa^{2} .
\end{aligned}
$$

Further, let there exist constants $\mu_{1}, \mu_{2}, \mu_{3}>0, \Lambda>0$, and some $0<\theta \leq 1$ such that for some $\lambda \in[0, \Lambda]$ the following two inequalities are satisfied for all $0<h<1 / \sqrt{\mu_{0}}$, all $0<\kappa<\widehat{\kappa}$ and all $m=1, \ldots, M:^{3}$

\footnotetext{
${ }^{3}$ Here we use the convention that for $\lambda=b_{m}=0$ we set $\left(\lambda+b_{m}\right)^{p-2} b_{m}^{2}=0$.
} 


$$
\begin{aligned}
& d_{t} a_{m}^{2}+\mu_{1}\left(\lambda+b_{m}\right)^{p-2} b_{m}^{2} \leq b_{m} r_{m}+b_{m} \rho_{m}+\mu_{2} b_{m-1} b_{m}+s_{m}^{2}+\sigma_{m}^{2}, \\
& d_{t} a_{m}^{2}+\mu_{1}\left(\lambda+b_{m}\right)^{p-2} b_{m}^{2} \leq b_{m} r_{m}+b_{m} \rho_{m}+\mu_{3} b_{m} b_{m-1}^{1-\theta} a_{m-1}^{\theta}+s_{m}^{2}+\sigma_{m}^{2} .
\end{aligned}
$$

Then, there exist constants $\overline{\mu_{0}}, \bar{\kappa}>0$, and $\mu_{4}, \mu_{5}>0$, independent of $\lambda$, such that for all $\kappa, h>0$ satisfying $\kappa<\bar{\kappa}$ and $h^{2}<\overline{\mu_{0}} \kappa$ there holds

$$
\begin{gathered}
\max _{0 \leq m \leq M} b_{m} \leq 1, \\
\max _{0 \leq m \leq M} a_{m}^{2}+\mu_{1}(1+\Lambda)^{p-2} \kappa \sum_{m=0}^{M} b_{m}^{2} \leq \mu_{4}\left(h^{2}+\kappa^{2}\right) \exp \left(2 \mu_{5} \kappa M\right) .
\end{gathered}
$$

Proof This result is a small modification of the corresponding results in [3, 5], and can be proved in the same way.

The following result will be used frequently in the sequel.

\section{Lemma 2.6 Assume that}

$$
f, \partial_{t} f \in L^{2}(I ; X)
$$

where $\left(X,\|\cdot\|_{X}\right)$ is a Banach space. Then, for all $\tau_{m} \in I_{m}, m=1, \ldots, M$, it holds

$$
\kappa \sum_{m=1}^{M} f_{I_{m}}\left\|f(s)-f\left(\tau_{m}\right)\right\|_{X}^{2} d s \leq \kappa^{2}\left\|\partial_{t} f\right\|_{L^{2}(I ; X)}^{2} .
$$

Proof The assertion is proved in [6, Lemma 3.1] in the special case $\tau_{m}=t_{m}$, $m=1, \ldots, M$. The general case follows exactly in the same way.

For the spatial discretization we denote by $\mathcal{T}_{h}$ a family of shape-regular triangulations, consisting of 3-dimensional closed simplices $K$. We denote by $h_{K}$ the diameter of $K$ and by $\rho_{K}$ the supremum of the diameters of inscribed balls. We assume that $\mathcal{T}_{h}$ is non-degenerate, i.e., there exists a constant $\gamma_{0}>0$ such that $\max _{K \in \mathcal{T}_{h}} \frac{h_{K}}{\rho_{K}} \leq \gamma_{0}$. The global mesh-size $h$ is defined by $h:=\max _{K \in \mathcal{T}_{h}} h_{K}$. Let $S_{K}$ denote the neighborhood of $K$, i.e., $S_{K}$ is the union of all simplices of $\mathcal{T}_{h}$ intersecting $K$. By the assumptions we obtain that $\left|S_{K}\right| \sim|K|$ and that the number of patches $S_{K}$ to which a simplex belongs are bounded uniformly in both $h>0$ and $K \in \mathcal{T}_{h}$.

We denote by $\mathcal{P}_{k}\left(\mathcal{T}_{h}\right)$, with $k \in \mathbb{N}_{0}:=\mathbb{N} \cup\{0\}$, the space of scalar or vector-valued functions, which are polynomials of degree at most $k$ on each $K \in \mathcal{T}_{h}$. Given a triangulation $\mathcal{T}_{h}$ of $\Omega$ with the above properties and given $r_{0}, r_{1}, s_{0} \in \mathbb{N}_{0}$, with $r_{0} \leq r_{1}$, we define

$$
X_{h}:=\left\{\mathbf{v}_{h} \in X \mid \mathbf{v}_{h} \in \mathcal{P}\right\} \quad \text { and } \quad Y_{h}:=\left\{\eta_{h} \in Y \mid \eta_{h} \in \mathcal{P}_{s_{0}}\left(\mathcal{T}_{h}\right)\right\},
$$

with $\mathcal{P}_{r_{0}}\left(\mathcal{T}_{h}\right) \subset \mathcal{P} \subseteq \mathcal{P}_{r_{1}}\left(\mathcal{T}_{h}\right)$. Note that there exists a constant $c=c\left(r_{1}, \gamma_{0}\right)$ such that for all $\mathbf{v}_{h} \in X_{h}, K \in \mathcal{T}_{h}, j \in \mathbb{N}_{0}$, and all $x \in K$ holds

$$
\left|\nabla^{j} \mathbf{v}_{h}(x)\right| \leq c \int_{K}\left|\nabla^{j} \mathbf{v}_{h}(y)\right| d y .
$$


For the weak formulation of discrete problems we use the following function spaces

$$
V_{h}:=V \cap X_{h}, \quad Q_{h}:=Q \cap Y_{h} .
$$

We also need some numerical interpolation operators. Rather than working with a specific interpolation operator we make the following assumptions:

Assumption 2.7 We assume that $r_{0}=1$ and that there exists a linear projection operator $\Pi_{h}^{\text {div }}: X \rightarrow X_{h}$ which

(a) is locally $W^{1,1}$-stable, i.e., for all $\mathbf{w} \in X$ and $K \in \mathcal{T}_{h}$ there holds

$$
f_{K}\left|\Pi_{h}^{\text {div }} \mathbf{w}\right| d x \leq c f_{S_{K}}|\mathbf{w}| d x+c f_{S_{K}} h_{K}|\nabla \mathbf{w}| d x ;
$$

(b) preserves zero boundary values, i.e., $\Pi_{h}^{\text {div }}(V) \subset V_{h}$;

(c) preserves divergence in the $Y_{h}^{*}$-sense, i.e., for all $\mathbf{w} \in X$ and $\eta_{h} \in Y_{h}$ there holds

$$
\left(\operatorname{div} \mathbf{w}, \eta_{h}\right)=\left(\operatorname{div} \Pi_{h}^{\operatorname{div}} \mathbf{w}, \eta_{h}\right) .
$$

Assumption 2.8 We assume that $Y_{h}$ contains the constant functions, i.e. that $\mathbb{R} \subset Y_{h}$, and that there exists a linear projection operator $\Pi_{h}^{Y}: Y \rightarrow Y_{h}$ which is locally $L^{1}$-stable, i.e., for all $q \in Y$ and $K \in \mathcal{T}_{h}$ there holds

$$
f_{K}\left|\Pi_{h}^{Y} q\right| d x \leq c f_{S_{K}}|q| d x .
$$

The existence of a projection operator $\Pi_{h}^{\text {div }}$ as in Assumption 2.7 is known (among others) for the Taylor-Hood, the Crouzeix-Raviart, and the MINI element in dimensions two and three; the Clément interpolation operator satisfies Assumption 2.8. For a discussion and consequences of these assumptions we refer to [2, Sec. 3.2], [6, Appendix], and [17, Sec. 4,5]. We collect in the next two propositions the properties of the projection operators, which are relevant for the present paper.

Proposition 2.9 Let $\Pi_{h}^{\text {div }}$ satisfy Assumption 2.7.

(i) Let $\mathbf{F}(\mathbf{D v}) \in W^{1,2}(\Omega)$. Then, there exists a constant $c=c\left(p, r_{1}, \gamma_{0}\right)$ such that

$$
\left\|\mathbf{F}(\mathbf{D v})-\mathbf{F}\left(\mathbf{D} \Pi_{h}^{\text {div }} \mathbf{v}\right)\right\|_{2} \leq c h\|\nabla \mathbf{F}(\mathbf{D v})\|_{2} .
$$

(ii) Let $r \in(1, \infty)$. Then, there exists a constant $c=c\left(r, r_{1}, \gamma_{0}\right)$ such that for all $\mathbf{v} \in W^{1, r}(\Omega)$ and all $\mathbf{w} \in W^{2, r}(\Omega)$ holds

$$
\begin{array}{r}
\left\|\mathbf{v}-\Pi_{h}^{\text {div }} \mathbf{v}\right\|_{r}+h\left\|\nabla \Pi_{h}^{\text {div }} \mathbf{v}\right\|_{r} \leq c h\|\nabla \mathbf{v}\|_{r}, \\
\left\|\nabla \mathbf{w}-\nabla \Pi_{h}^{\operatorname{div}} \mathbf{w}\right\|_{r} \leq c h\left\|\nabla^{2} \mathbf{w}\right\|_{r} .
\end{array}
$$

(iii) Let $r \in[1,2]$ and let $\ell=1$ or $\ell=2$ be such that $W^{\ell, r}(\Omega) \hookrightarrow \hookrightarrow L^{2}(\Omega)$. Then, there exists a constant $c=c\left(r, \ell, r_{1}, \gamma_{0}\right)$ such that for all $\mathbf{v} \in W^{\ell, r}(\Omega)$ holds

$$
\left\|\mathbf{v}-\Pi_{h}^{\text {div }} \mathbf{v}\right\|_{2} \leq c h^{\ell+3\left(\frac{1}{2}-\frac{1}{r}\right)}\left\|\nabla^{\ell} \mathbf{v}\right\|_{r} .
$$


(iv) Let $\mathbf{F}(\mathbf{D v}) \in W^{1,2}(\Omega)$ and $\mathbf{F}(\mathbf{D w}) \in L^{2}(\Omega)$. Then, there exists a constant $c=$ $c\left(p, r_{1}, \gamma_{0}\right)$ such that

$$
\int_{\Omega} \varphi_{|\mathbf{D v}|}\left(\left|\mathbf{D} \Pi_{h}^{\mathrm{div}} \mathbf{v}-\mathbf{D} \Pi_{h}^{\mathrm{div}} \mathbf{w}\right|\right) d x \leq c h^{2}\|\nabla \mathbf{F}(\mathbf{D v})\|_{2}^{2}+c\|\mathbf{F}(\mathbf{D v})-\mathbf{F}(\mathbf{D w})\|_{2}^{2} .
$$

Proof The first two assertions are proved, e.g., in [2, 17]. The last two assertions are proved in [6].

Proposition 2.10 Let $\Pi_{h}^{Y}$ satisfy Assumption 2.8. Let $\psi$ be an $\mathrm{N}$-function satisfying the $\Delta_{2}$ condition. Then, there exists a constant $c=c\left(\gamma_{0}, \Delta_{2}(\psi)\right)$ such that for all sufficiently smooth functions and all $K \in \mathcal{T}_{h}$ there holds

$$
\begin{gathered}
\int_{K} \psi\left(\left|\Pi_{h}^{Y} q\right|\right) d x \leq c \int_{S_{K}} \psi(|q|) d x, \\
\int_{K} \psi\left(\left|q-\Pi_{h}^{Y} q\right|\right) d x \leq c \int_{S_{K}} \psi\left(h_{K}|\nabla q|\right) d x .
\end{gathered}
$$

Proof The assertions are proved in [17].

\subsection{Main results}

Before we formulate the main result, proving optimal convergence rates for the error between the solution $\mathbf{u}$ of the continuous problem (1.1) and the discrete solution $\left\{\mathbf{u}_{h}^{m}\right\}_{m=0}^{M}$ of the space-time discretization (1.4), we discuss the existence of solutions of (1.1) and (1.4).

The existence of global weak solutions $\mathbf{u} \in L^{\infty}\left(I ; L^{2}(\Omega)\right) \cap L^{p}\left(I ; V_{\text {div }}\right)$ of (1.1) for large data is proved in [18] for $\delta \geq 0$ and $p>\frac{6}{5}$. The existence of a locally in time, unique strong solution $\quad \mathbf{u} \in L^{r}\left(I^{\prime} ; W^{2, r}(\Omega)\right) \cap L^{p}\left(I^{\prime} ; V_{\mathrm{div}}\right) \cap W^{1, r}\left(I^{\prime} ; L^{r}(\Omega)\right), \quad q \in L^{r}\left(I^{\prime} ; W^{1, r}(\Omega)\right) \cap$ $L^{r}\left(I^{\prime} ; L_{0}^{r}(\Omega)\right)$ for any $r \in(5, \infty)$ and some $I^{\prime}:=\left(0, T^{\prime}\right)$ with $T^{\prime} \in(0, T)$ of (1.1) for large data is proved in [8] for $\delta>0$ and $p>1$.

The existence of a unique discrete solution $\left\{\mathbf{u}_{h}^{m}\right\}_{m=1}^{M},\left\{q_{h}^{m}\right\}_{m=1}^{M}$ can be inferred in the following way. Setting $V_{h}(0):=\left\{\mathbf{w}_{h} \in V_{h} \mid\left(\operatorname{div} \mathbf{w}_{h}, \eta_{h}\right)=0 \forall \eta_{h} \in Y_{h}\right\}$, one uses Brouwer's fixed point theorem and the properties of the extra stress tensor $\mathbf{S}$ and the convective term to show the existence of $\mathbf{u}_{h}^{m} \in V_{h}(0)$ satisfying (1.4) for all $\mathbf{v}_{h} \in V_{h}(0)$ (cf. [20, Lemma 7.1]). This solution is unique due to the semi-implicit discretization of the convective term and the monotonicity of $\mathbf{S}$. Moreover, testing (1.4) with $\left\{\mathbf{u}_{h}^{m}\right\}_{m=1}^{M}$ yields the energy estimate

$$
\max _{m=1, \ldots, M}\left\|\mathbf{u}_{h}^{m}\right\|_{2}^{2}+\kappa \sum_{m=1}^{M}\left\|\mathbf{F}\left(\mathbf{D} \mathbf{u}_{h}^{m}\right)\right\|_{2}^{2} \leq C\left(\mathbf{u}_{0}, \mathbf{f}\right) .
$$

The properties of the projection operators $\Pi_{h}^{\text {div }}$ and $\Pi_{h}^{Y}$ ensure the validity of the discrete inf-sup condition (cf. [2, Lemma 4.1]). Since the divergence is a closed, surjective, linear operator from $V_{h}$ onto $Q_{h}$ and since the gradient is the annihilator of the kernel of the 
divergence, these ensure the existence of a unique $q_{h}^{m} \in Q_{h}$ such that $\left\{\mathbf{u}_{h}^{m}\right\}_{m=1}^{M},\left\{q_{h}^{m}\right\}_{m=1}^{M}$ satisfy (1.4).

Now we can formulate our main result.

Theorem 2.11 Let the extra stress tensor $\mathbf{S}$ in (1.1) have $(p, \delta)$-structure for some $p \in\left(\frac{6}{5}, 2\right]$, and some $\delta \in[0, \infty)$ fixed but arbitrary. Let $\Omega \subset \mathbb{R}^{3}$ be a bounded polyhedral domain with Lipschitz continuous boundary, and $I=(0, T), T \in(0, \infty)$, be a finite time interval. Assume that $\mathbf{f} \in W^{1,2}\left(I ; L^{2}(\Omega)\right), \mathbf{u}_{0} \in W^{2, p}(\Omega) \cap V_{\mathrm{div}}$ and that the solution $(\mathbf{u}, q)$ of (1.1) satisfies (1.2) and

$$
\mathbf{F}(\mathbf{D u}) \in W^{1,2}(I \times \Omega) \quad \text { and } \quad q \in L^{p^{\prime}}\left(I ; L_{0}^{p^{\prime}}(\Omega) \cap W^{1, p^{\prime}}(\Omega)\right) .
$$

Let the space $V_{h}, h>0$, be defined as above with $r_{0}=1$ and let $\left\{\mathbf{u}_{h}^{m}\right\}_{m=1}^{M},\left\{q_{h}^{m}\right\}_{m=1}^{M}$ be the unique solution of (1.4). Then, for $p \in\left(\frac{8}{5}, 2\right]$ there exists $\kappa_{0} \in(0,1]$ such that, for given $h \in(0,1)$ and $\kappa \in\left(0, \kappa_{0}\right)$ satisfying

$$
h^{4 / p^{\prime}} \leq \sigma_{0} \kappa
$$

for some $\sigma_{0}>0$, the following error estimate holds true

$$
\max _{m=1, \ldots, M}\left\|\mathbf{u}_{h}^{m}-\mathbf{u}\left(t_{m}\right)\right\|_{2}^{2}+\kappa \sum_{m=1}^{M}\left\|\mathbf{F}\left(\mathbf{D} \mathbf{u}_{h}^{m}\right)-\mathbf{F}\left(\mathbf{D u}\left(t_{m}\right)\right)\right\|_{2}^{2} \leq c\left(h^{2}+\kappa^{2}\right),
$$

with a constant $c$ depending only on the characteristics of $\mathbf{S},\|\mathbf{F}(\mathbf{D u})\|_{W^{1,2}(I \times \Omega)}$, $\left\|\partial_{t} \mathbf{f}\right\|_{L^{2}\left(I ; L^{2}(\Omega)\right.},\left\|\mathbf{u}_{0}\right\|_{2, p},\|\nabla q\|_{L^{p^{\prime}(I \times \Omega)}}, \gamma_{0}, r_{1}, \delta$, and $\sigma_{0}$.

\subsection{Comparison with previous results and observation on the requested regularity}

Here we compare the new result with previous ones on the discretization of generalized non-Newtonian fluids (and general parabolic equations and systems). We also discuss briefly the regularity we are assuming on the continuous solution.

Concerning previously proved error-estimates we can mainly recall the following facts:

(i) Problem (1.1), in the case of space periodic boundary conditions, has been treated in $[5,14,15,20]$. In [5] the same optimal error estimate as in Theorem 2.11 is proved under slightly stronger assumptions on the regularity of the solution $\mathbf{u}$ of $(1.1)$, for $p \in\left(\frac{3}{2}, 2\right]$.

Problem (1.1) without the convective term $[\nabla \mathbf{u}] \mathbf{u}$, in the case of homogeneous Dirichlet boundary conditions, has been treated in [19] for $p \in\left(\frac{6}{5}, \infty\right)$. It is shown there that (2.16) holds with the right-hand side replaced by $c\left(h^{\min \left\{2, \frac{4}{p}\right\}}+\kappa^{2}\right)$. The proofs of these results are based on intermediate semi-discrete problems, for which a certain regularity has to be proved, to obtain the desired optimal convergence rates. This in fact limits the results in $[5,14,15,20]$ to the case of space periodic boundary conditions. Here, we avoid such (technical) problems by proving the error estimate directly without using intermediate semi-discrete problems. The same perspective is also taken in the recent papers $[6,11]$. In [11] problem (1.1) is treated for a nonlinear operator $\mathbf{S}$ depending on the full gradient $\nabla \mathbf{u}$, having $(p(\cdot, \cdot), \delta)$-structure, with a variable exponent $p(\cdot, \cdot)$, but without convective term and without the solenoidality condition. In this situation the error estimate (2.16) with the 
right-hand side replaced by $c\left(h^{2 \alpha_{x}}+\kappa^{2 \alpha_{t}}\right)$ is proved if the variable exponent belongs to $C^{\alpha_{x}, \alpha_{t}}(\overline{I \times \Omega})$ for appropriate $\alpha_{x}, \alpha_{t} \in(0,1]$ and an additional CFL-condition $\kappa^{r} \leq c \inf _{K \in \mathcal{T}_{h} h_{k}}$, with some $r \geq \frac{1+2 \alpha_{t}}{2 \alpha_{x}}$, is satisfied. In [6] problem (1.1) is treated without convective term and without the solenoidality condition. There the error estimate (2.16) is proved under the same conditions as in Theorem 2.11.

(ii) In the recent paper [9] a different approach is used to treat the unsteady $p$-Laplace problem, i.e., problem (1.1) without convective term and without the solenoidality constraint. By using the $L^{2}$-projection operator instead of the Scott-Zhang operator (cf. [22]), satisfying Assumption 2.7, the error estimate

$$
\max _{m=1, \ldots, M}\left\|\mathbf{u}_{h}^{m}-f_{t_{m-1}}^{t_{m+1}} \mathbf{u}(s) d s\right\|_{2}^{2}+\kappa \sum_{m=1}^{M} \int_{t_{m-1}}^{t_{m+1}}\left\|\mathbf{F}\left(\mathbf{D} \mathbf{u}_{h}^{m}\right)-\mathbf{F}(\mathbf{D u}(s))\right\|_{2}^{2} d s \leq c\left(h^{2 \alpha_{s}}+\kappa^{2 \alpha_{t}}\right),
$$

is proved in [9] without a coupling condition between $h$ and $\kappa$. The removal of the coupling is due to the usage of the $L^{2}$-projection which in the treatment of the time derivative does not produce terms needing a coupling [cf. estimate (3.4)]. However, the treatment of the nonlinear operator $\mathbf{S}$ is subtle and requires delicate estimates, which result in the different error estimate (2.17) compared to the error estimate (2.16). The estimate (2.17) is proved under the assumption that

$$
\begin{aligned}
\mathbf{F}(\nabla \mathbf{u}) & \in L^{2}\left(I ; N^{\alpha_{x}, 2}(\Omega)\right) \cap N^{\alpha_{t}, 2}\left(I ; L^{2}(\Omega)\right) \\
\mathbf{u} & \in L^{2}\left(I ; N^{\alpha_{x}, 2}(\Omega)\right)
\end{aligned}
$$

for $\frac{1}{2}<\alpha_{t} \leq 1$ and $0<\alpha_{x} \leq 1$, where $N^{\alpha, 2}$ are appropriate Nikol'skiř spaces with differentiability $\alpha \in(0,1]$ (cf. [9]). Even for $\alpha_{x}=\alpha_{t}=1$ estimate (2.17) differs from (2.16) since it contains time averages of the error rather than a point-wise error. The usage of limited regularity in the time-variable and time averaging of the error is motivated by a similar analysis performed for stochastic parabolic equations in [10].

(iii) In $[23,24]$ the convergence of a fully implicit space-time discretization (without convergence rate but also with no assumptions of smoothness of the limiting problem) of the problem (1.1) in the case of homogeneous Dirichlet boundary conditions is proved for $p>\frac{11}{5}$ and even for $p>\frac{6}{5}$ for a regularized problem. The convergence of the same numerical scheme (1.4) towards a weak solution has been recently proved in [7] for $p>\frac{11}{5}$. In fact, in [7] the convergence of a general quasi non-conforming Rothe-Galerkin scheme in the context of evolution problems with Bochner pseudo-monotone operators is proved (cf. [1] for the treatment of a conforming Rothe-Galerkin scheme in the context of evolution problems with Bochner pseudo-monotone operators).

Let us now discuss the natural regularity assumption (2.14). The assumption $(2.14)_{1}$ is natural in the sense that it is the one obtained from the extra stress tensor $\mathbf{S}$ if formally tested with $-\Delta \mathbf{u}$ and $\partial_{t}^{2} \mathbf{u}$. The existence of solutions satisfying (2.14) $)_{1}$ is proved rigorously for problem (1.1) in the case of periodic boundary conditions locally in time in [4, 16], for $p>\frac{7}{5}$ and large data. The situation for Dirichlet boundary conditions is more complicated. The existence of a locally in time unique strong solution 


$$
\begin{aligned}
& \mathbf{u} \in L^{r}\left(I^{\prime} ; W^{2, r}(\Omega)\right) \cap L^{p}\left(I^{\prime} ; V_{\mathrm{div}}\right) \cap W^{1, r}\left(I^{\prime} ; L^{r}(\Omega)\right), \\
& q \in L^{r}\left(I^{\prime} ; W^{1, r}(\Omega)\right) \cap L^{r}\left(I^{\prime} ; L_{0}^{r}(\Omega)\right)
\end{aligned}
$$

for any $r \in(5, \infty)$ and some $I^{\prime}:=\left(0, T^{\prime}\right)$ with $T^{\prime} \in(0, T)$ is proved in [8] for large data. This regularity implies, using parabolic embedding theory (cf. [16, Appendix]), that $\mathbf{F}(\mathbf{D u}) \in L^{2}\left(I^{\prime} ; W^{1,2}(\Omega)\right)$ and that $\mathbf{u}, \nabla \mathbf{u} \in C\left(\overline{I^{\prime} \times \Omega}\right)$. However, in [8] it is not proved that this solution also satisfies $\partial_{t} \mathbf{F}(\mathbf{D u}) \in L^{2}\left(I^{\prime} ; L^{2}(\Omega)\right)$. Nevertheless, one can show that the solution from [8] also satisfies $\partial_{t} \mathbf{F}(\mathbf{D u}) \in L^{2}\left(I^{\prime} ; L^{2}(\Omega)\right)$, using the following auxiliary result.

Proposition 2.12 Let the extra stress tensor $\mathbf{S}$ in (1.1) have $(p, \delta)$-structure for some $p \in\left(\frac{6}{5}, 2\right]$, and some $\delta \in[0, \infty)$. Let $\Omega \subset \mathbb{R}^{d}, d \geq 2$, be a bounded domain with Lipschitz continuous boundary, and $I=(0, T), T \in(0, \infty)$, be a finite time interval. Assume that $\mathbf{u}_{0} \in V_{\text {div }} \quad$ satisfies $\quad \operatorname{div} \mathbf{S}\left(\mathbf{D u}_{0}\right) \in L^{2}(\Omega), \quad \mathbf{f} \in L^{p^{\prime}}\left(I ; L^{p^{\prime}}(\Omega)\right) \cap W^{1,2}\left(I ; L^{2}(\Omega)\right) \quad$ and $\mathbf{G} \in C\left(\bar{I} ; W^{1,2}(\Omega)\right) \cap W^{1, p^{\prime}}\left(I ; L^{p^{\prime}}(\Omega)\right)$. Then, there exists a unique weak solution $\mathbf{v} \in$ $L^{\infty}\left(I ; L^{2}(\Omega)\right) \cap L^{p}\left(I ; V_{\text {div }}\right)$ of

$$
\begin{aligned}
\partial_{t} \mathbf{v}-\operatorname{div} \mathbf{S}(\mathbf{D} \mathbf{v})+\nabla q & =\mathbf{f}+\operatorname{div} \mathbf{G} & & \text { in } I \times \Omega, \\
\operatorname{div} \mathbf{v} & =0 & & \text { in } I \times \Omega, \\
\mathbf{v}(0) & =\mathbf{u}_{0} & & \text { in } \Omega,
\end{aligned}
$$

satisfying additionally $\partial_{t} \mathbf{v} \in L^{\infty}\left(I ; L^{2}(\Omega)\right)$ and $\partial_{t} \mathbf{F}(\mathbf{D v}) \in L^{2}\left(I ; L^{2}(\Omega)\right)$.

Proof This result is proved using ideas from $[4,16]$. Using the Galerkin method and the theory of monotone operators one shows that there exists a unique weak solutions $\mathbf{v} \in$ $L^{\infty}\left(I ; L^{2}(\Omega)\right) \cap L^{p}\left(I ; V_{\text {div }}\right)$ of (2.18). Moreover, the regularity of the data allows us to show that we can take the time derivative of the Galerkin equations and test with the time derivative of the Galerkin solution. Straightforward manipulations show that this produces an estimate, showing after a limiting process in the Galerkin parameter, the additional regularity stated above.

Next, by using Proposition 2.12 with $\mathbf{G}=\mathbf{u} \otimes \mathbf{u}$ (where $\mathbf{u}$ is the solution from [8]), the monotonicity of $\mathbf{S}$, and the above regularity for $\mathbf{u}$ imply that the solution from [8] satisfies also $\partial_{t} \mathbf{F}(\mathbf{D u}) \in L^{2}\left(I^{\prime} ; L^{2}(\Omega)\right)$. Consequently, the unique solution $(\mathbf{u}, q)$ from [8] satisfies (2.14) with $I$ replaced by $I^{\prime}$.

It is useful to formulate the consequences of the assumption $\mathbf{F}(\mathbf{D u}) \in W^{1,2}(I \times \Omega)$ in terms of Bochner-Sobolev spaces. From [16, Thm. 33] and standard embedding results it follows that

$$
\mathbf{F}(\mathbf{D u}) \in C\left(\bar{I} ; L^{3}(\Omega)\right) .
$$

Using $|\mathbf{D u}|^{p / 2}+\delta^{\frac{p}{2}} \sim|\mathbf{F}(\mathbf{D u})|+\delta^{\frac{p}{2}}$ and the continuity of $\mathbf{P} \mapsto \mathbf{F}^{-1}(\mathbf{P})$ (cf. [4, Lemma 3.23]) we get

$$
\mathbf{u} \in C\left(\bar{I} ; W^{1,3 p / 2}(\Omega)\right) .
$$

In [4, Lemma 4.5] it is shown that for $p \leq 2$ 


$$
\begin{gathered}
\left\|\nabla^{2} \mathbf{u}\right\|_{\frac{6 p}{4+p}}^{2} \leq c\|\nabla \mathbf{F}(\mathbf{D u})\|_{2}^{2}\left(\delta+\|\nabla \mathbf{u}\|_{3 p / 2}\right)^{2-p}, \\
\left\|\partial_{t} \nabla \mathbf{u}\right\|_{\frac{6 p}{4+p}}^{2} \leq c\left\|\partial_{t} \mathbf{F}(\mathbf{D u})\right\|_{2}^{2}\left(\delta+\|\nabla \mathbf{u}\|_{3 p / 2}\right)^{2-p} .
\end{gathered}
$$

Thus, we also get

$$
\begin{gathered}
\mathbf{u} \in L^{2}\left(I ; W^{2 \frac{6 p}{4+p}}(\Omega)\right), \\
\partial_{t} \mathbf{u} \in L^{2}\left(I ; W^{1 \frac{6 p}{4+p}}(\Omega)\right),
\end{gathered}
$$

where the bounds depend only on $\|\mathbf{F}(\mathbf{D u})\|_{W^{1,2}(I \times \Omega)}$ and $\delta_{0}$.

Finally we would like to comment on the restriction $p \in\left(\frac{8}{5}, 2\right]$ in Theorem 2.11 compared to the restriction $p \in\left(\frac{3}{2}, 2\right]$ in [5, Theorem 2.6]. Based on the results from [4] it is assumed in [5] that, additionally to $(2.14)_{1}$, the solution satisfies among other properties $\mathbf{F}(\mathbf{D u}) \in L^{2 \frac{5 p-6}{2-p}}\left(I ; W^{1,2}(\Omega)\right)$. This results in

$$
\mathbf{u} \in C\left(\bar{I} ; W^{1, r}(\Omega)\right) \quad \text { for any } r \in[1,6(p-1)) .
$$

If we also assume that (2.21) holds, we can improve Theorem 2.11 to the range $p \in\left(\frac{3}{2}, 2\right]$. More precisely, we have:

Corollary 2.13 Assume that in the situation of Theorem 2.11 the solution $\mathbf{u}$ of (1.1) additionally satisfies (2.21). Then, the error estimate (2.16) holds for $p \in\left(\frac{3}{2}, 2\right]$ with a constant additionally depending on $\|\mathbf{u}\|_{C\left(\bar{I} ; W^{1, r}(\Omega)\right)}$, for some suitable $r \in[1,6(p-1))$.

\section{Proof of the main result}

In this section we prove the error estimates from Theorem 2.11. To this end we need to derive an equation for the error and to use the discrete Gronwall Lemma 2.5 together with approximation properties due to the regularity of the solution and the properties of the extra stress tensor $\mathbf{S}$.

In the error equation we want to use the test function $\mathbf{u}_{h}^{m}-\Pi_{h}^{\text {div }} \mathbf{u}\left(t_{m}\right)$, which belongs to the space $V_{h}(0)$. Thus, it is enough to consider test functions $\mathbf{v}_{h}$ from $V_{h}(0)$ in (1.4). For such test functions we can replace the discrete pressure $q_{h}^{m}$ by an arbitrary function from $Q_{h}$. Thus, it follows from (1.4) $)_{1}$ that for all $\mathbf{v}_{h} \in V_{h}(0), \mu_{h} \in Q_{h}$ and $m=1, \ldots, M$ there holds

$$
\left(d_{t} \mathbf{u}_{h}^{m}, \mathbf{v}_{h}\right)+\left(\mathbf{S}\left(\mathbf{D} \mathbf{u}_{h}^{m}\right), \mathbf{D} \mathbf{v}_{h}\right)+b\left(\mathbf{u}_{h}^{m-1}, \mathbf{u}_{h}^{m}, \mathbf{v}_{h}\right)-\left(\mu_{h}, \operatorname{div} \mathbf{v}_{h}\right)=\left(\mathbf{f}\left(t_{m}\right), \mathbf{v}_{h}\right) .
$$

We can choose in (3.1), for each $m=1, \ldots, M$,

$$
\mu_{h}=\mu_{h}^{m}:=\int_{I_{m}} \Pi_{h}^{Y} q(t) d t
$$

and since $\left.\underset{I_{m}}{(f} \Pi_{h}^{Y} q(t) d t, \operatorname{div} \mathbf{v}_{h}\right)=\underset{I_{m}}{f}\left(\Pi_{h}^{Y} q(t), \operatorname{div} \mathbf{v}_{h}\right) d t$, we get for all $\mathbf{v}_{h} \in V_{h}(0)$ and $m=1, \ldots, M$, that there holds

$$
\left(d_{t} \mathbf{u}_{h}^{m}, \mathbf{v}_{h}\right)+\left(\mathbf{S}\left(\mathbf{D} \mathbf{u}_{h}^{m}\right), \mathbf{v}_{h}\right)+b\left(\mathbf{u}_{h}^{m-1}, \mathbf{u}_{h}^{m}, \mathbf{v}_{h}\right)-f_{I_{m}}\left(\Pi_{h}^{Y} q(t), \operatorname{div} \mathbf{v}_{h}\right) d t=\left(\mathbf{f}\left(t_{m}\right), \mathbf{v}_{h}\right),
$$


which we can re-write also as follows:

$$
\begin{aligned}
& \left(d_{t} \mathbf{u}_{h}^{m}, \mathbf{v}_{h}\right)+f_{I_{m}}\left(\mathbf{S}\left(\mathbf{D} \mathbf{u}_{h}^{m}\right), \mathbf{v}_{h}\right) d t+f_{I_{m}} b\left(\mathbf{u}_{h}^{m-1}, \mathbf{u}_{h}^{m}, \mathbf{v}_{h}\right) d t-f_{I_{m}}\left(\Pi_{h}^{Y} q(t), \operatorname{div} \mathbf{v}_{h}\right) d t \\
& =f_{I_{m}}\left(\mathbf{f}\left(t_{m}\right), \mathbf{v}_{h}\right) d t
\end{aligned}
$$

since we are averaging locally constant terms. We subtract from this equation the retarded averages over $I_{m}$ of Eq. (1.2) and obtain the equation for the error

$$
\begin{aligned}
& \left(d_{t}\left(\mathbf{u}_{h}^{m}-\mathbf{u}\left(t_{m}\right)\right), \mathbf{v}_{h}\right)+f_{I_{m}}\left(\mathbf{S}\left(\mathbf{D} \mathbf{u}_{h}^{m}\right)-\mathbf{S}(\mathbf{D u}(t)), \mathbf{D} \mathbf{v}_{h}\right) d t \\
& \quad+f_{I_{m}} b\left(\mathbf{u}_{h}^{m-1}, \mathbf{u}_{h}^{m}, \mathbf{v}_{h}\right)-b\left(\mathbf{u}(t), \mathbf{u}(t), \mathbf{v}_{h}\right) d t-f_{I_{m}}\left(\Pi_{h}^{Y} q(t)-q(t), \operatorname{div} \mathbf{v}_{h}\right) d t \\
& =f_{I_{m}}\left(\mathbf{f}\left(t_{m}\right)-\mathbf{f}(t), \mathbf{v}_{h}\right) d t,
\end{aligned}
$$

valid for all $m=1, \ldots, M$ and all $\mathbf{v}_{h} \in V_{h}(0)$.

Choosing now the legitimate test function $\mathbf{v}_{h}=\mathbf{u}_{h}^{m}-\Pi_{h}^{\text {div }} \mathbf{u}\left(t_{m}\right) \in V_{h}(0)$ we finally get for all $m=1, \ldots, M$

$$
\begin{aligned}
& \left(d_{t}\left(\mathbf{u}_{h}^{m}-\mathbf{u}\left(t_{m}\right)\right), \mathbf{u}_{h}^{m}-\Pi_{h}^{\mathrm{div}} \mathbf{u}\left(t_{m}\right)\right) \\
& \quad+f_{I_{m}}\left(\mathbf{S}\left(\mathbf{D} \mathbf{u}_{h}^{m}\right)-\mathbf{S}(\mathbf{D} \mathbf{u}(t)), \mathbf{D} \mathbf{u}_{h}^{m}-\mathbf{D} \Pi_{h}^{\mathrm{div}} \mathbf{u}\left(t_{m}\right)\right) d t \\
& \quad+f_{I_{m}} b\left(\mathbf{u}_{h}^{m-1}, \mathbf{u}_{h}^{m}, \mathbf{u}_{h}^{m}-\Pi_{h}^{\mathrm{div}} \mathbf{u}\left(t_{m}\right)\right)-b\left(\mathbf{u}(t), \mathbf{u}(t), \mathbf{u}_{h}^{m}-\Pi_{h}^{\mathrm{div}} \mathbf{u}\left(t_{m}\right)\right) d t \\
& \quad-f_{I_{m}}\left(\Pi_{h}^{Y} q(t)-q(t), \operatorname{div} \mathbf{u}_{h}^{m}-\operatorname{div} \Pi_{h}^{\operatorname{div}} \mathbf{u}\left(t_{m}\right)\right) d t \\
& =\underset{I_{m}}{f}\left(\mathbf{f}\left(t_{m}\right)-\mathbf{f}(t), \mathbf{u}_{h}^{m}-\Pi_{h}^{\operatorname{div}} \mathbf{u}\left(t_{m}\right)\right) d t .
\end{aligned}
$$

We now discuss and estimate the terms in (3.3) separately, to arrive finally to the estimate (3.22).

First, note that the projection operator $\Pi_{h}^{\text {div }}$ has the same properties as the operator $P_{h}$ considered in [6] and that the solution $\mathbf{u}$ of (1.2) and the solution treated in [6] possess exactly the same regularity. Thus, the first two terms on the left-hand side can be treated exactly as in [6]. Consequently, [6, Lemmas 3.7, 3.9] yield the following estimates: 


$$
\begin{aligned}
& \left(d_{t}\left(\mathbf{u}_{h}^{m}-\mathbf{u}\left(t_{m}\right)\right), \mathbf{u}_{h}^{m}-\Pi_{h}^{\mathrm{div}} \mathbf{u}\left(t_{m}\right)\right) \\
& \geq \frac{1}{2} d_{t}\left\|\mathbf{u}_{h}^{m}-\mathbf{u}\left(t_{m}\right)\right\|_{2}^{2}-c \frac{h^{2+4 / p^{\prime}}}{\kappa} \int_{I_{m}}\left\|\nabla^{2} \mathbf{u}(t)\right\|_{\frac{6 p}{4+p}}^{2} d t \\
& -c \frac{h^{4 / p^{\prime}}}{\kappa}\left\|\nabla \mathbf{u}\left(t_{m}\right)-f_{I_{m}} \nabla \mathbf{u}(t) d t\right\|_{\frac{6 p}{4+p}}^{2},
\end{aligned}
$$

and

$$
\begin{aligned}
f_{I_{m}}\left(\mathbf{S}\left(\mathbf{D u} \mathbf{u}_{h}^{m}\right)-\mathbf{S}(\mathbf{D u}(t)), \mathbf{D} \mathbf{u}_{h}^{m}-\mathbf{D} \Pi_{h}^{\mathrm{div}} \mathbf{u}\left(t_{m}\right)\right) d t \\
\quad \geq\left\|\mathbf{F}\left(\mathbf{D} \mathbf{u}_{h}^{m}\right)-\mathbf{F}\left(\mathbf{D u}\left(t_{m}\right)\right)\right\|_{2}^{2}-c h^{2} f_{I_{m}}\|\nabla \mathbf{F}(\mathbf{D u}(t))\|_{2}^{2} d t \\
\quad-c f_{I_{m}}\left\|\mathbf{F}(\mathbf{D u}(t))-\mathbf{F}\left(\mathbf{D u}\left(t_{m}\right)\right)\right\|_{2}^{2} d t .
\end{aligned}
$$

The term with the external force is treated slightly differently compared to [6, Lemma 3.10]. This is due to the fact that to apply Gronwall's Lemma 2.5 we need an estimate involving the $L^{p}$-norm of the gradient of the error. To shorten the notation in the following computations we denote the error for $m=1, \ldots, M$ by

$$
\mathbf{e}_{h}^{m}:=\mathbf{u}_{h}^{m}-\mathbf{u}\left(t_{m}\right) \text {. }
$$

Lemma 3.1 Under the hypotheses of Theorem 2.11 we have

$$
\begin{aligned}
& \left|f_{I_{m}}\left(\mathbf{f}\left(t_{m}\right)-\mathbf{f}(t), \mathbf{u}_{h}^{m}-\Pi_{h}^{\mathrm{div}} \mathbf{u}\left(t_{m}\right)\right) d t\right| \\
& \leq c \int_{I_{m}}\left\|\mathbf{f}\left(t_{m}\right)-\mathbf{f}(t)\right\|_{2}^{2} d t+c\left\|\mathbf{D e}_{h}^{m}\right\|_{p} f_{I_{m}}\left\|\mathbf{f}\left(t_{m}\right)-\mathbf{f}(t)\right\|_{2} d t \\
& \quad+c h^{2+4 / p^{\prime}} f_{I_{m}}\left\|\nabla^{2} \mathbf{u}(t)\right\|_{\frac{6 p}{p+4}}^{2} d t+c h^{4 / p^{\prime}} \int_{I_{m}}\left\|\nabla \mathbf{u}\left(t_{m}\right)-\nabla \mathbf{u}(t)\right\|_{\frac{6 p p}{4+p}}^{2} d t .
\end{aligned}
$$

Proof Using that

$$
\mathbf{u}_{h}^{m}-\Pi_{h}^{\mathrm{div}} \mathbf{u}\left(t_{m}\right)=\mathbf{u}_{h}^{m}-\mathbf{u}\left(t_{m}\right)+\mathbf{u}\left(t_{m}\right)-\Pi_{h}^{\mathrm{div}} \mathbf{u}\left(t_{m}\right)=\mathbf{e}_{h}^{m}+\mathbf{u}\left(t_{m}\right)-\Pi_{h}^{\mathrm{div}} \mathbf{u}\left(t_{m}\right),
$$

together with Hölder's inequality, Young's inequality and the embedding $W^{1, p}(\Omega) \hookrightarrow L^{2}(\Omega)$, valid for $p \geq \frac{6}{5}$, we get 


$$
\begin{aligned}
& \left|f_{I_{m}}\left(\mathbf{f}\left(t_{m}\right)-\mathbf{f}(t), \mathbf{u}_{h}^{m}-\Pi_{h}^{\mathrm{div}} \mathbf{u}\left(t_{m}\right)\right) d t\right| \\
& \leq c\left\|\mathbf{D} \mathbf{e}_{h}^{m}\right\|_{p} \int_{I_{m}}\left\|\mathbf{f}\left(t_{m}\right)-\mathbf{f}(t)\right\|_{2} d t \\
& \quad+c f_{I_{m}}\left\|\mathbf{f}\left(t_{m}\right)-\mathbf{f}(t)\right\|_{2}^{2} d t+c\left\|\mathbf{u}\left(t_{m}\right)-\Pi_{h}^{\mathrm{div}} \mathbf{u}\left(t_{m}\right)\right\|_{2}^{2} .
\end{aligned}
$$

The last term was already treated in the proof of [6, Lemma 3.7], where it is proved that

$$
\begin{aligned}
& \left\|\mathbf{u}\left(t_{m}\right)-\Pi_{h}^{\mathrm{div}} \mathbf{u}\left(t_{m}\right)\right\|_{2}^{2} \\
& \leq c h^{2+4 / p^{\prime}} \int_{I_{m}}\left\|\nabla^{2} \mathbf{u}(t)\right\|_{\frac{\sigma p}{p+4}}^{2} d t+c h^{4 / p^{\prime}} f_{I_{m}}\left\|\nabla \mathbf{u}\left(t_{m}\right)-\nabla \mathbf{u}(t)\right\|_{\frac{6 p}{4+p}}^{2} d t,
\end{aligned}
$$

which yields the assertion.

It remains to treat the convective term and the pressure term, which were not present in [6] and which require a precise estimation. Let us start with the former one.

Lemma 3.2 Under the hypotheses of Theorem 2.11 we have

$$
\begin{aligned}
& \left|\int_{I_{m}} b\left(\mathbf{u}_{h}^{m-1}, \mathbf{u}_{h}^{m}, \mathbf{u}_{h}^{m}-\Pi_{h}^{\mathrm{div}} \mathbf{u}\left(t_{m}\right)\right)-b\left(\mathbf{u}(t), \mathbf{u}(t), \mathbf{u}_{h}^{m}-\Pi_{h}^{\mathrm{div}} \mathbf{u}\left(t_{m}\right)\right) d t\right| \\
& \leq c\left\|\mathbf{D} \mathbf{e}_{h}^{m}\right\|_{p}\left\|\mathbf{D} \mathbf{e}_{h}^{m-1}\right\|_{p}^{1-\theta}\left\|\mathbf{e}_{h}^{m-1}\right\|_{2}^{\theta}+c\left\|\mathbf{D} \mathbf{e}_{h}^{m}\right\|_{p} \int_{I_{m}}\left\|\nabla \mathbf{u}\left(t_{m}\right)-\nabla \mathbf{u}(t)\right\|_{\frac{6 p}{4+p}} d t \\
& \quad+c\left\|\mathbf{D} \mathbf{e}_{h}^{m}\right\|_{p} f_{I_{m}}\left\|\nabla \mathbf{u}(t)-\nabla \mathbf{u}\left(t_{m-1}\right)\right\|_{\frac{6 p}{4+p}} d t+c\left\|\mathbf{D e}_{h}^{m}\right\|_{p} h f_{I_{m}}\left\|\nabla^{2} \mathbf{u}(t)\right\|_{\frac{6 p}{4+p}} d t .
\end{aligned}
$$

Moreover, the estimate is also correct if the first term on the right-hand side is replaced by $c\left\|\mathbf{D} \mathbf{e}_{h}^{m}\right\|_{p}\left\|\mathbf{D e}_{h}^{m-1}\right\|_{p}$.

Proof Since $\Pi_{h}^{\text {div }} \mathbf{u}_{h}^{m}=\mathbf{u}_{h}^{m}$, we get $\mathbf{u}_{h}^{m}-\Pi_{h}^{\text {div }} \mathbf{u}\left(t_{m}\right)=\Pi_{h}^{\text {div }} \mathbf{e}_{h}^{m}$. Thus, we can re-write the integrand in the term to be estimated in (3.6) as follows

$$
b\left(\mathbf{u}_{h}^{m-1}, \mathbf{u}_{h}^{m}, \Pi_{h}^{\text {div }} \mathbf{e}_{h}^{m}\right)-b\left(\mathbf{u}(t), \mathbf{u}(t), \Pi_{h}^{\text {div }} \mathbf{e}_{h}^{m}\right) .
$$

To the latter we add and subtract, in the order, the terms $b\left(\mathbf{u}_{h}^{m-1}, \Pi_{h}^{\text {div }} \mathbf{u}\left(t_{m}\right), \Pi_{h}^{\text {div }} \mathbf{e}_{h}^{m}\right)$, $b\left(\mathbf{u}\left(t_{m-1}\right), \Pi_{h}^{\text {div }} \mathbf{u}\left(t_{m}\right), \Pi_{h}^{\text {div }} \mathbf{e}_{h}^{m}\right), b\left(\mathbf{u}\left(t_{m-1}\right), \mathbf{u}\left(t_{m}\right), \Pi_{h}^{\text {div }} \mathbf{e}_{h}^{m}\right)$ and $b\left(\mathbf{u}(t), \mathbf{u}\left(t_{m}\right), \Pi_{h}^{\text {div }} \mathbf{e}_{h}^{m}\right)$, to get for all $m=1, \ldots, M$ and a.e. $t \in I_{m}$

$$
\begin{aligned}
& b\left(\mathbf{u}_{h}^{m-1}, \mathbf{u}_{h}^{m}, \Pi_{h}^{\mathrm{div}} \mathbf{e}_{h}^{m}\right)-b\left(\mathbf{u}(t), \mathbf{u}(t), \Pi_{h}^{\mathrm{div}} \mathbf{e}_{h}^{m}\right)=b\left(\mathbf{u}_{h}^{m-1}, \mathbf{u}_{h}^{m}-\Pi_{h}^{\mathrm{div}} \mathbf{u}\left(t_{m}\right), \Pi_{h}^{\mathrm{div}} \mathbf{e}_{h}^{m}\right) \\
& \quad+b\left(\mathbf{u}_{h}^{m-1}-\mathbf{u}\left(t_{m-1}\right), \Pi_{h}^{\mathrm{div}} \mathbf{u}\left(t_{m}\right), \Pi_{h}^{\mathrm{div}} \mathbf{e}_{h}^{m}\right)+b\left(\mathbf{u}\left(t_{m-1}\right), \Pi_{h}^{\mathrm{div}} \mathbf{u}\left(t_{m}\right)-\mathbf{u}\left(t_{m}\right), \Pi_{h}^{\mathrm{div}} \mathbf{e}_{h}^{m}\right) \\
& \quad+b\left(\mathbf{u}\left(t_{m-1}\right)-\mathbf{u}(t), \mathbf{u}\left(t_{m}\right), \Pi_{h}^{\mathrm{div}} \mathbf{e}_{h}^{m}\right)+b\left(\mathbf{u}(t), \mathbf{u}\left(t_{m}\right)-\mathbf{u}(t), \Pi_{h}^{\mathrm{div}} \mathbf{e}_{h}^{m}\right) \\
& =: I_{1}^{m}(t)+I_{2}^{m}(t)+I_{3}^{m}(t)+I_{4}^{m}(t)+I_{5}^{m}(t) .
\end{aligned}
$$


In view of the skew-symmetry of $b(\cdot, \cdot, \cdot)$ we have $I_{1}^{m}(t)=0$, for all $m=1, \ldots, M$ and $t \in I_{m}$.

Using the definition of $b(\cdot, \cdot, \cdot)$ in (1.3), and partial integration we get for all $m=$ $1, \ldots, M$ and $t \in I_{m}$, also using Hölder's inequality with $\left(\frac{3 p}{2}, \frac{3 p}{4 p-5}, \frac{3 p}{3-p}\right)$ and $\left(p, \frac{3 p}{4 p-5}, \frac{3 p}{2-p}\right)$, respectively, the embeddings $W^{1, p}(\Omega) \hookrightarrow L^{\frac{3 p}{3-p}}(\Omega), W^{1, \frac{3 p}{2}}(\Omega) \hookrightarrow L^{\frac{3 p}{2-p}}(\Omega)$, Korn's inequality, the interpolation of $L^{\frac{3 p}{4 p-5}}(\Omega)$ between $L^{2}(\Omega)$ and $W^{1, p}(\Omega)$, which is possible for $p \in\left(\frac{8}{5}, 2\right]$, the continuity of $\Pi_{h}^{\text {div }}$ (cf. Proposition 2.9 (ii)), and (2.19), that

$$
\begin{aligned}
\left|I_{2}^{m}(t)\right| & \leq \frac{1}{2}\left|\left(\left[\nabla \Pi_{h}^{\mathrm{div}} \mathbf{u}\left(t_{m}\right)\right] \mathbf{e}_{h}^{m-1}, \Pi_{h}^{\mathrm{div}} \mathbf{e}_{h}^{m}\right)\right|+\frac{1}{2}\left|\left(\left[\nabla \Pi_{h}^{\mathrm{div}} \mathbf{e}_{h}^{m}\right] \mathbf{e}_{h}^{m-1}, \Pi_{h}^{\mathrm{div}} \mathbf{u}\left(t_{m}\right)\right)\right| \\
& \leq c\left\|\nabla \Pi_{h}^{\mathrm{div}} \mathbf{u}\left(t_{m}\right)\right\|_{\frac{3 p}{2}}\left\|\mathbf{e}_{h}^{m-1}\right\|_{\frac{3 p}{4 p-5}}\left\|\mathbf{D} \Pi_{h}^{\mathrm{div}} \mathbf{e}_{h}^{m}\right\|_{p} \\
& \leq c\left\|\mathbf{e}_{h}^{m-1}\right\|_{2}^{\theta}\left\|\mathbf{D} \mathbf{e}_{h}^{m-1}\right\|_{p}^{1-\theta}\left\|\mathbf{D} \mathbf{e}_{h}^{m}\right\|_{p},
\end{aligned}
$$

with $\theta:=\frac{10 p-16}{5 p-6} \in(0,1]$ for $p \in\left(\frac{8}{5}, 2\right]$. Using the embedding $W^{1, p}(\Omega) \hookrightarrow L^{2}(\Omega)$ in the last line we also obtain

$$
\left|I_{2}^{m}(t)\right| \leq c\left\|\mathbf{D} \mathbf{e}_{h}^{m-1}\right\|_{p}\left\|\mathbf{D} \mathbf{e}_{h}^{m}\right\|_{p} .
$$

Since $\mathbf{u}\left(t_{m-1}\right)$ is solenoidal we get, also using Hölder's inequality with $\left(\frac{3 p}{5(p-1)}, \frac{3 p}{2-p}, \frac{3 p}{3-p}\right)$, the embeddings $W^{1, p}(\Omega) \hookrightarrow L^{\frac{3 p}{3-p}}(\Omega), W^{1 \frac{3 p}{2}}(\Omega) \hookrightarrow L^{\frac{3 p}{2-p}}(\Omega), L^{\frac{6 p}{4+p}}(\Omega) \hookrightarrow L^{\frac{3 p}{(p-1)}}(\Omega)$, the continuity of $\Pi_{h}^{\text {div }}$ (cf. Proposition 2.9 (ii)), and (2.19), that

$$
\begin{aligned}
\left|I_{3}^{m}(t)\right| & =\left|\left(\left[\nabla \Pi_{h}^{\mathrm{div}} \mathbf{u}\left(t_{m}\right)-\nabla \mathbf{u}\left(t_{m}\right)\right] \mathbf{u}\left(t_{m-1}\right), \Pi_{h}^{\mathrm{div}} \mathbf{e}_{h}^{m}\right)\right| \\
& \leq c\left\|\nabla \Pi_{h}^{\mathrm{div}} \mathbf{u}\left(t_{m}\right)-\nabla \mathbf{u}\left(t_{m}\right)\right\|_{\frac{3 p}{5(p-1)}}\left\|\nabla \mathbf{u}\left(t_{m}\right)\right\|_{\frac{3 p}{2}}\left\|\mathbf{D} \Pi_{h}^{\mathrm{div}} \mathbf{e}_{h}^{m}\right\|_{p} \\
& \leq c\left\|\nabla \Pi_{h}^{\mathrm{div}} \mathbf{u}\left(t_{m}\right)-\nabla \mathbf{u}\left(t_{m}\right)\right\|_{\frac{{ }_{0} p}{4+p}}\left\|\mathbf{D} \mathbf{e}_{h}^{m}\right\|_{p} .
\end{aligned}
$$

To treat $\left\|\nabla \Pi_{h}^{\text {div }} \mathbf{u}\left(t_{m}\right)-\nabla \mathbf{u}\left(t_{m}\right)\right\|_{\frac{6 p}{4+p}}$ we add and subtract $\nabla \Pi_{h}^{\text {div }}\left(f_{I_{m}} \mathbf{u}(\sigma) d \sigma\right)$, use $\Pi_{h}^{\text {div }}\left(\int_{I_{m}} \mathbf{u}(\sigma) d \sigma\right)=f_{I_{m}} \Pi_{h}^{\text {div }} \mathbf{u}(\sigma) d \sigma$, add and subtract $\nabla \underset{I_{m}}{f} \mathbf{u}(\sigma) d \sigma$, use the continuity of $\Pi_{h}^{\text {div }}$ (cf. Proposition 2.9 (ii)), $\nabla \underset{I_{m}}{f} \mathbf{v}(\sigma) d \sigma=f_{I_{m}} \nabla \nabla \mathbf{v}(\sigma) d \sigma$, Fubini's theorem, the properties of the Bochner integral, Proposition 2.9 (ii) and (2.20) to arrive at

$$
\begin{aligned}
& \left.\| \nabla \Pi_{h}^{\mathrm{div}} \mathbf{u}\left(t_{m}\right)-\nabla \mathbf{u}\left(t_{m}\right)\right) \|_{\frac{6 p}{4+p}} \\
& \leq c\left\|\nabla \Pi_{h}^{\mathrm{div}} f_{I_{m}} \mathbf{u}\left(t_{m}\right)-\mathbf{u}(\sigma) d \sigma\right\|_{\frac{6 p}{4+p}}+c\left\|\nabla f_{I_{m}} \Pi_{h}^{\mathrm{div}} \mathbf{u}(\sigma)-\mathbf{u}(\sigma) d \sigma\right\|_{\frac{\sigma p}{4+p}} \\
& +c\left\|\nabla f_{I_{m}} \mathbf{u}(\sigma)-\mathbf{u}\left(t_{m}\right) d \sigma\right\|_{\frac{\sigma_{p}}{4+p}} \\
& \leq c\left\|\int_{I_{m}} \nabla \mathbf{u}\left(t_{m}\right)-\nabla \mathbf{u}(\sigma) d \sigma\right\|_{\frac{\sigma p}{4+p}}+c\left\|\int_{I_{m}} \nabla \Pi_{h}^{\mathrm{div}} \mathbf{u}(\sigma)-\nabla \mathbf{u}(\sigma) d \sigma\right\|_{\frac{\sigma p}{4+p}} \\
& \leq c \int_{I_{m}}\left\|\nabla \mathbf{u}\left(t_{m}\right)-\nabla \mathbf{u}(\sigma)\right\|_{\frac{6 p}{4+p}} d \sigma+c f_{I_{m}}\left\|\nabla \Pi_{h}^{\mathrm{div}} \mathbf{u}(\sigma)-\nabla \mathbf{u}(\sigma)\right\|_{\frac{6 p}{4+p}} d \sigma \\
& \leq c f_{I_{m}}\left\|\nabla \mathbf{u}\left(t_{m}\right)-\nabla \mathbf{u}(\sigma)\right\|_{\frac{\sigma_{p}}{4+p}} d \sigma+c h f_{I_{m}}\left\|\nabla^{2} \mathbf{u}(\sigma)\right\|_{\frac{\sigma_{p}}{4+p}} d \sigma .
\end{aligned}
$$


Combining (3.10) and (3.11) we showed that for all $m=1, \ldots, M$ and $t \in I_{m}$ there holds

$$
\begin{aligned}
\left|I_{3}^{m}(t)\right| \leq & c\left\|\mathbf{D} \mathbf{e}_{h}^{m}\right\|_{p} f_{I_{m}}\left\|\nabla \mathbf{u}\left(t_{m}\right)-\nabla \mathbf{u}(\sigma)\right\|_{\frac{6 p}{4+p}} d \sigma \\
& +c\left\|\mathbf{D} \mathbf{e}_{h}^{m}\right\|_{p} h f_{I_{m}}\left\|\nabla^{2} \mathbf{u}(\sigma)\right\|_{\frac{6 p}{4+p}} d \sigma .
\end{aligned}
$$

Since $\mathbf{u}\left(t_{m-1}\right)$ and $\mathbf{u}(t)$ are solenoidal we get for every $m=1, \ldots, M$ and $t \in I_{m}$, also using Hölder's inequality with $\left(\frac{3 p}{2}, \frac{3 p}{4 p-5}, \frac{3 p}{3-p}\right)$, the embedding $W^{1, p}(\Omega) \hookrightarrow L^{\frac{3 p}{3-p}}(\Omega)$, Korn's inequality, the embedding $W^{1 \frac{6 p}{4+p}}(\Omega) \hookrightarrow L^{\frac{6 p}{4-p}}(\Omega) \hookrightarrow L^{\frac{3 p}{4 p-5}}(\Omega)$, the continuity of $\Pi_{h}^{\text {div }}$ (cf. Proposition 2.9 (ii)), and (2.19), that

$$
\begin{aligned}
\left|I_{4}^{m}(t)\right| & =\left|\left(\left[\nabla \mathbf{u}\left(t_{m}\right)\right]\left(\mathbf{u}(t)-\mathbf{u}\left(t_{m-1}\right)\right), \Pi_{h}^{\mathrm{div}} \mathbf{e}_{h}^{m}\right)\right| \\
& \leq c\left\|\nabla \mathbf{u}\left(t_{m}\right)\right\|_{\frac{3 p}{2}}\left\|\mathbf{u}(t)-\mathbf{u}\left(t_{m}\right)\right\|_{\frac{3 p}{4 p-5}}\left\|\mathbf{D} \Pi_{h}^{\mathrm{div}} \mathbf{e}_{h}^{m}\right\|_{p} \\
& \leq c\left\|\nabla \mathbf{u}(t)-\nabla \mathbf{u}\left(t_{m-1}\right)\right\|_{\frac{6 p}{4+p}}\left\|\mathbf{D} \mathbf{e}_{h}^{m}\right\|_{p} .
\end{aligned}
$$

Since $\mathbf{u}(t)$ is solenoidal we get for every $m=1, \ldots, M$ and $t \in I_{m}$, also using Hölder's inequality with $\left(\frac{6 p}{4+p}, \frac{6 p}{7 p-10}, \frac{3 p}{3-p}\right)$, the embedding $W^{1, p}(\Omega) \hookrightarrow L^{\frac{3 p}{3-p}}(\Omega)$, Korn's inequality, the embedding $W^{1, \frac{3 p}{2}}(\Omega) \hookrightarrow L^{\frac{3 p}{2-p}}(\Omega) \hookrightarrow L^{\frac{6 p}{7 p-10}}(\Omega),(2.19)$, and the continuity of $\Pi_{h}^{\text {div }}$ (cf. Proposition 2.9 (ii)), that

$$
\begin{aligned}
\left|I_{5}^{m}(t)\right| & =\left|\left(\left[\nabla \mathbf{u}\left(t_{m}\right)-\nabla \mathbf{u}(t)\right] \mathbf{u}(t), \Pi_{h}^{\mathrm{div}} \mathbf{e}_{h}^{m}\right)\right| \\
& \leq c\left\|\nabla \mathbf{u}\left(t_{m}\right)-\nabla \mathbf{u}(t)\right\|_{\frac{6 p}{4+p}}\|\mathbf{u}(t)\|_{\frac{6 p}{7 p-10}}\left\|\mathbf{D} \Pi_{h}^{\mathrm{div}} \mathbf{e}_{h}^{m}\right\|_{p} \\
& \leq c\left\|\nabla \mathbf{u}\left(t_{m}\right)-\nabla \mathbf{u}(t)\right\|_{\frac{6 p}{4+p}}\left\|\mathbf{D} \mathbf{e}_{h}^{m}\right\|_{p} .
\end{aligned}
$$

The assertions follow from (3.8) and (3.9), resp., (3.12), (3.13) and (3.14).

Let us now discuss the last term, namely the one including the pressure.

Lemma 3.3 Under the hypotheses of Theorem 2.11 for every $\varepsilon>0$ there exists $c_{\varepsilon}>0$ such that

$$
\begin{aligned}
& \left|f_{I_{m}}\left(\Pi_{h}^{Y} q(t)-q(t), \operatorname{div} \mathbf{u}_{h}^{m}-\operatorname{div} \Pi_{h}^{\operatorname{div}} \mathbf{u}\left(t_{m}\right)\right) d t\right| \\
& \leq \varepsilon\left\|\mathbf{F}\left(\mathbf{D} \mathbf{u}_{h}^{m}\right)-\mathbf{F}\left(\mathbf{D u}\left(t_{m}\right)\right)\right\|_{2}^{2}+c_{\varepsilon} \int_{I_{m}}\left\|\mathbf{F}\left(\mathbf{D u}\left(t_{m}\right)\right)-\mathbf{F}(\mathbf{D u}(t))\right\|_{2}^{2} d t \\
& \quad+c_{\varepsilon} f_{I_{m}}\left\|\mathbf{F}(\mathbf{D u}(t))-\mathbf{F}\left(\mathbf{D} \Pi_{h}^{\operatorname{div}} \mathbf{u}(t)\right)\right\|_{2}^{2} d t+c_{\varepsilon} h^{2} f_{I_{m}}\|\nabla \mathbf{F}(\mathbf{D u}(t))\|_{2}^{2} d t \\
& \quad+c_{\varepsilon} h^{2} f_{I_{m}}\|\nabla q(t)\|_{p^{\prime}}^{p^{\prime}}+\|\mathbf{F}(\mathbf{D u}(t))\|_{2}^{2} d t+c_{\varepsilon} h^{2}|\Omega| \delta^{p^{\prime}} .
\end{aligned}
$$


Proof The point-wise application of Young's inequality (2.4) with $a=\left|\mathbf{D u}\left(t_{m}, \cdot\right)\right|$ to the integrand of the term on the left-hand side of (3.15) yields for all $m=1, \ldots, M$, a.e. $t \in I_{m}$ and every $\varepsilon>0$

$$
\begin{aligned}
& \left|\left(\Pi_{h}^{Y} q(t)-q(t), \operatorname{div} \mathbf{u}_{h}^{m}-\operatorname{div} \Pi_{h}^{\operatorname{div}} \mathbf{u}\left(t_{m}\right)\right)\right| \\
& \leq \frac{\varepsilon}{c_{0}} \int_{\Omega} \varphi_{\left|\mathbf{D u}\left(t_{m}\right)\right|}\left(\left|\mathbf{D} \mathbf{u}_{h}^{m}-\mathbf{D} \Pi_{h}^{\operatorname{div}} \mathbf{u}\left(t_{m}\right)\right|\right) d x \\
& \quad+c_{\varepsilon} \int_{\Omega}\left(\varphi_{\left|\mathbf{D u}\left(t_{m}\right)\right|}\right)^{*}\left(\left|\Pi_{h}^{Y} q(t)-q(t)\right|\right) d x \leq \varepsilon\left\|\mathbf{F}\left(\mathbf{D} \mathbf{u}_{h}^{m}\right)-\mathbf{F}\left(\mathbf{D u}\left(t_{m}\right)\right)\right\|_{2}^{2}+\varepsilon \| \mathbf{F}\left(\mathbf{D u}\left(t_{m}\right)\right) \\
& \quad-\mathbf{F}\left(\mathbf{D} \Pi_{h}^{\operatorname{div}} \mathbf{u}\left(t_{m}\right)\right) \|_{2}^{2}+c_{\varepsilon} \int_{\Omega}\left(\varphi_{\left|\mathbf{D u}\left(t_{m}\right)\right|}\right)^{*}\left(\left|\Pi_{h}^{Y} q(t)-q(t)\right|\right) d x \\
& =: \varepsilon J_{1}^{m}(t)+\varepsilon J_{2}^{m}(t)+c_{\varepsilon} J_{3}^{m}(t),
\end{aligned}
$$

where in the last estimate we added and subtracted $\mathbf{D u}\left(t_{m}\right)$ and used that for all $\mathbf{P}, \mathbf{Q} \in$ $\mathbb{R}_{\text {sym }}^{3 \times 3}$ there holds $\varphi_{|\mathbf{P}|}(|\mathbf{P}-\mathbf{Q}|) \leq c_{0}|\mathbf{F}(\mathbf{P})-\mathbf{F}(\mathbf{Q})|^{2}$ (cf. Proposition 2.3 (i)). To treat $J_{2}^{m}(t)$ we add and subtract $\mathbf{F}(\mathbf{D u}(t))$ and $\mathbf{F}\left(\mathbf{D} \Pi_{h}^{\text {div }} \mathbf{u}(t)\right)$

$$
\begin{aligned}
\left|J_{2}^{m}(t)\right| \leq & c\left\|\mathbf{F}\left(\mathbf{D u}\left(t_{m}\right)\right)-\mathbf{F}(\mathbf{D u}(t))\right\|_{2}^{2}+\left\|\mathbf{F}(\mathbf{D u}(t))-\mathbf{F}\left(\mathbf{D} \Pi_{h}^{\operatorname{div}} \mathbf{u}(t)\right)\right\|_{2}^{2} \\
& +\left\|\mathbf{F}\left(\mathbf{D} \Pi_{h}^{\operatorname{div}} \mathbf{u}(t)\right)-\mathbf{F}\left(\mathbf{D} \Pi_{h}^{\operatorname{div}} \mathbf{u}\left(t_{m}\right)\right)\right\|_{2}^{2} .
\end{aligned}
$$

To treat the last term on the right-hand side of (3.17) we use Proposition 2.3 and Proposition 2.9 (iv) to get

$$
\begin{aligned}
& \left\|\mathbf{F}\left(\mathbf{D} \Pi_{h}^{\mathrm{div}} \mathbf{u}(t)\right)-\mathbf{F}\left(\mathbf{D} \Pi_{h}^{\mathrm{div}} \mathbf{u}\left(t_{m}\right)\right)\right\|_{2}^{2} \\
& \leq c \int_{\Omega} \varphi_{\left|\mathbf{D} \Pi_{h}^{\mathrm{div}} \mathbf{u}(t)\right|}\left(\left|\mathbf{D} \Pi_{h}^{\mathrm{div}} \mathbf{u}(t)-\mathbf{D} \Pi_{h}^{\mathrm{div}} \mathbf{u}\left(t_{m}\right)\right|\right) d x \\
& \leq c \int_{\Omega} \varphi_{|\mathbf{D u}(t)|}\left(\left|\mathbf{D} \Pi_{h}^{\mathrm{div}} \mathbf{u}(t)-\mathbf{D} \Pi_{h}^{\mathrm{div}} \mathbf{u}\left(t_{m}\right)\right|\right) d x+c\left\|\mathbf{F}\left(\mathbf{D} \Pi_{h}^{\mathrm{div}} \mathbf{u}(t)\right)-\mathbf{F}(\mathbf{D u}(t))\right\|_{2}^{2} \\
& \leq c h^{2}\|\nabla \mathbf{F}(\mathbf{D u}(t))\|_{2}^{2}+c\left\|\mathbf{F}(\mathbf{D u}(t))-\mathbf{F}\left(\mathbf{D u}\left(t_{m}\right)\right)\right\|_{2}^{2} \\
& \quad+c\left\|\mathbf{F}\left(\mathbf{D} \Pi_{h}^{\mathrm{div}} \mathbf{u}(t)\right)-\mathbf{F}(\mathbf{D u}(t))\right\|_{2}^{2} .
\end{aligned}
$$

To treat the term $J_{3}^{m}(t)$ we note that $\Omega=\bigcup_{K \in \mathcal{T}_{h}} K$, use Proposition 2.3 (ii), (iii) and $K \subseteq S_{K}$ to arrive at

$$
\begin{aligned}
\left|J_{3}^{m}(t)\right| \leq & c \sum_{K \in \mathcal{T}_{h}} \int_{K}\left(\varphi_{\left.\left|\left\langle\mathbf{D u}\left(t_{m}\right)\right\rangle_{S_{K}}\right|\right)^{*}\left(\left|\Pi_{h}^{Y} q(t)-q(t)\right|\right) d x}\right. \\
& +c \sum_{K \in \mathcal{T}_{h}} \int_{S_{K}}\left|\mathbf{F}\left(\mathbf{D u}\left(t_{m}\right)\right)-\left\langle\mathbf{F}\left(\mathbf{D u}\left(t_{m}\right)\right)\right\rangle_{S_{K}}\right|^{2} d x \\
= & : c \sum_{K \in \mathcal{T}_{h}} A_{K}^{m}(t)+c \sum_{K \in \mathcal{T}_{h}} B_{K}^{m}(t) .
\end{aligned}
$$


Using Proposition 2.10, again Proposition $2.3 \quad$ (ii), (iii) and $\left(\varphi_{|\mathbf{D u}(t)|}\right)^{*}(h|\sigma|) \leq c h^{2}\left(|\sigma|^{p^{\prime}}+\delta^{p^{\prime}}+|\mathbf{F}(\mathbf{D u}(t))|^{2}\right)$, valid for $p \leq 2$, and $h_{K} \leq h$ yields

$$
\begin{aligned}
\left|A_{K}^{m}(t)\right| \leq & \int_{S_{K}}\left(\varphi_{\left|\left\langle\mathbf{D u}\left(t_{m}\right)\right\rangle_{S_{K}}\right|}\right)^{*}\left(h_{K}|\nabla q(t)|\right) d x \\
\leq & c \int_{S_{K}}\left(\varphi_{\left|\mathbf{D u}\left(t_{m}\right)\right|}\right)^{*}\left(h_{K}|\nabla q(t)|\right) d x+c B_{K}^{m}(t) \\
\leq & c \int_{S_{K}}\left(\varphi_{|\mathbf{D u}(t)|}\right)^{*}\left(h_{K}|\nabla q(t)|\right) d x \\
& +\int_{S_{K}}\left|\mathbf{F}\left(\mathbf{D u}\left(t_{m}\right)\right)-\mathbf{F}(\mathbf{D u}(t))\right|^{2} d x+c B_{K}^{m}(t) . \\
\leq & c h^{2} \int_{S_{K}}|\nabla q(t)|^{p^{\prime}}+\delta^{p^{\prime}}+c|\mathbf{F}(\mathbf{D u}(t))|^{2} d x \\
& +\int_{S_{K}}\left|\mathbf{F}\left(\mathbf{D u}\left(t_{m}\right)\right)-\mathbf{F}(\mathbf{D u}(t))\right|^{2} d x+c B_{K}^{m}(t) .
\end{aligned}
$$

Adding and subtracting appropriate terms, using Proposition 2.3 (iii), the properties of the mean value, $\left|S_{K}\right| \sim|K|$ and Poincaré's inequality we get

$$
\begin{aligned}
\left|B_{K}^{m}(t)\right| \leq & c \int_{S_{K}}\left|\mathbf{F}\left(\mathbf{D u}\left(t_{m}\right)\right)-\mathbf{F}(\mathbf{D u}(t))\right|^{2} d x+c \int_{S_{K}}\left|\mathbf{F}(\mathbf{D u}(t))-\langle\mathbf{F}(\mathbf{D u}(t))\rangle_{S_{K}}\right|^{2} d x \\
& +c \int_{S_{K}}\left|\langle\mathbf{F}(\mathbf{D u}(t))\rangle_{S_{K}}-\left\langle\mathbf{F}\left(\mathbf{D u}\left(t_{m}\right)\right)\right\rangle_{S_{K}}\right|^{2} d x \\
\leq & c \int_{S_{K}}\left|\mathbf{F}\left(\mathbf{D u}\left(t_{m}\right)\right)-\mathbf{F}(\mathbf{D u}(t))\right|^{2} d x+c h^{2} \int_{S_{K}}|\nabla \mathbf{F}(\mathbf{D u}(t))|^{2} d x .
\end{aligned}
$$

The assertion follows from (3.16)-(3.21) and the properties of the triangulation.

Collecting all estimates, we are ready to prove the main result of this paper.

Proof of Theorem 2.11 From estimates (3.4) and (3.5), Lemmas 3.1, 3.2, and 3.3 we get, choosing $\varepsilon>0$ sufficiently small to absorb the term $\varepsilon\left\|\mathbf{F}\left(\mathbf{D u} \mathbf{u}_{h}^{m}\right)-\mathbf{F}\left(\mathbf{D u}\left(t_{m}\right)\right)\right\|_{2}^{2}$, and using the properties of the retarded time averages, that for all $m=1, \ldots, M$ and $0<\kappa \leq 1$ 


$$
\begin{aligned}
& d_{t}\left\|\mathbf{u}_{h}^{m}-\mathbf{u}\left(t_{m}\right)\right\|_{2}^{2}+c\left\|\mathbf{F}\left(\mathbf{D} \mathbf{u}_{h}^{m}\right)-\mathbf{F}\left(\mathbf{D u}\left(t_{m}\right)\right)\right\|_{2}^{2} \\
& \leq c h^{2} f_{I_{m}}\|\nabla \mathbf{F}(\mathbf{D u}(t))\|_{2}^{2} d t+c \frac{h^{2+4 / p^{\prime}}}{\kappa} \int_{I_{m}}\left\|\nabla^{2} \mathbf{u}(t)\right\|_{\frac{6 p}{4+p}}^{2} d t+c h^{2} f_{I_{m}}\|\nabla q(t)\|_{p^{\prime}}^{p^{\prime}} d t \\
& +c \int_{I_{m}}\left\|\mathbf{F}(\mathbf{D u}(t))-\mathbf{F}\left(\mathbf{D u}\left(t_{m}\right)\right)\right\|_{2}^{2} d t+c \frac{h^{4 / p^{\prime}}}{\kappa} f_{I_{m}}\left\|\nabla \mathbf{u}\left(t_{m}\right)-\nabla \mathbf{u}(t)\right\|_{\frac{6 p}{4+p}}^{2} d t \\
& +c f_{I_{m}}\left\|\mathbf{F}(\mathbf{D u}(t))-\mathbf{F}\left(\mathbf{D} \Pi_{h}^{\mathrm{div}} \mathbf{u}(t)\right)\right\|_{2}^{2} d t+c h^{2} f_{I_{m}}\|\mathbf{F}(\mathbf{D u}(t))\|_{2}^{2} d t \\
& +c h^{2}|\Omega| \delta^{p^{\prime}}+c f_{I_{m}}\left\|\mathbf{f}\left(t_{m}\right)-\mathbf{f}(t)\right\|_{2}^{2} d t+c\left\|\mathbf{D e}_{h}^{m}\right\|_{p} f_{I_{m}}\left\|\mathbf{f}\left(t_{m}\right)-\mathbf{f}(t)\right\|_{2} d t \\
& +c\left\|\mathbf{D} \mathbf{e}_{h}^{m}\right\|_{p}\left\|\mathbf{D} \mathbf{e}_{h}^{m-1}\right\|_{p}^{1-\theta}\left\|\mathbf{e}_{h}^{m-1}\right\|_{2}^{\theta}+c\left\|\mathbf{D} \mathbf{e}_{h}^{m}\right\|_{p} f_{I_{m}}\left\|\nabla \mathbf{u}\left(t_{m}\right)-\nabla \mathbf{u}(t)\right\|_{\frac{6 p}{4+p}} d t \\
& +c\left\|\mathbf{D} \mathbf{e}_{h}^{m}\right\|_{p} h f_{I_{m}}\left\|\nabla^{2} \mathbf{u}(t)\right\|_{\frac{{ }_{0} p}{4+p}} d t+c\left\|\mathbf{D} \mathbf{e}_{h}^{m}\right\|_{p} f_{I_{m}}\left\|\nabla \mathbf{u}(t)-\nabla \mathbf{u}\left(t_{m-1}\right)\right\|_{\frac{6 p}{4+p}} d t .
\end{aligned}
$$

Moreover, the estimate (3.22) is also correct if $c\left\|\mathbf{D} \mathbf{e}_{h}^{m}\right\|_{p}\left\|\mathbf{D} \mathbf{e}_{h}^{m-1}\right\|_{p}^{1-\theta}\left\|\mathbf{e}_{h}^{m-1}\right\|_{2}^{\theta}$ is replaced by $c\left\|\mathbf{D} \mathbf{e}_{h}^{m}\right\|_{p}\left\|\mathbf{D} \mathbf{e}_{h}^{m-1}\right\|_{p}$. To use Lemma 2.5 we observe that by Lemma 2.4 and (2.19) we have with $\lambda:=\delta+\|\mathbf{D u}\|_{C\left(\bar{I} ; L^{p}(\Omega)\right)}$

$$
\begin{aligned}
\left\|\mathbf{F}\left(\mathbf{D} \mathbf{u}_{h}^{m}\right)-\mathbf{F}\left(\mathbf{D u}\left(t_{m}\right)\right)\right\|_{2}^{2} & \geq c\left(\lambda+\left\|\mathbf{D} \mathbf{u}_{h}^{m}-\mathbf{D u}\left(t_{m}\right)\right\|_{p}\right)^{p-2}\left\|\mathbf{D} \mathbf{u}_{h}^{m}-\mathbf{D u}\left(t_{m}\right)\right\|_{p}^{2} \\
& =c\left(\lambda+\left\|\mathbf{D} \mathbf{e}_{h}^{m}\right\|_{p}\right)^{p-2}\left\|\mathbf{D} \mathbf{e}_{h}^{m}\right\|_{p}^{2} .
\end{aligned}
$$

Thus, the left-hand side of (3.22) is larger or equal than

$$
d_{t}\left\|\mathbf{e}_{h}^{m}\right\|_{2}^{2}+c\left(\lambda+\left\|\mathbf{D} \mathbf{e}_{h}^{m}\right\|_{p}\right)^{p-2}\left\|\mathbf{D} \mathbf{e}_{h}^{m}\right\|_{p}^{2},
$$

and (3.22) is now written in the form needed for the application of Lemma 2.5. To do so, we set

$$
\begin{aligned}
& a_{m}(h):=\left\|\mathbf{e}_{h}^{m}\right\|_{2}, \quad b_{m}(h):=\left\|\mathbf{D} \mathbf{e}_{h}^{m}\right\|_{p}, \quad r_{m}(h, \kappa):=h \int_{I_{m}}\left\|\nabla^{2} \mathbf{u}(t)\right\|_{\frac{g_{p}}{4+p}} d t, \\
& \rho_{m}(h, \kappa)=\rho_{m}:=\int_{I_{m}}\left\|\nabla \mathbf{u}\left(t_{m}\right)-\nabla \mathbf{u}(t)\right\|_{\frac{6_{p}}{4+p}} d t+\int_{I_{m}}\left\|\nabla \mathbf{u}(t)-\nabla \mathbf{u}\left(t_{m-1}\right)\right\|_{\frac{\sigma_{p}}{4+p}} d t \\
& +\int_{I_{m}}\left\|\mathbf{f}\left(t_{m}\right)-\mathbf{f}(t)\right\|_{2} d t \\
& \left(s_{m}(h, \kappa)\right)^{2}:=h^{2} f_{I_{m}}\|\nabla \mathbf{F}(\mathbf{D u}(t))\|_{2}^{2} d t+\frac{h^{2+4 / p^{\prime}}}{\kappa} f_{I_{m}}\left\|\nabla^{2} \mathbf{u}(t)\right\|_{\frac{6 p}{4+p}}^{2} d t \\
& +\int_{I_{m}}\left\|\mathbf{F}(\mathbf{D u}(t))-\mathbf{F}\left(\mathbf{D} \Pi_{h}^{\mathrm{div}} \mathbf{u}(t)\right)\right\|_{2}^{2} d t+h^{2} \int_{I_{m}}\|\nabla q(t)\|_{p^{\prime}}^{p^{\prime}} d t \\
& +h^{2} \int_{I_{m}}\|\mathbf{F}(\mathbf{D u}(t))\|_{2}^{2} d t+h^{2}|\Omega| \delta^{p^{\prime}}, \\
& \left(\sigma_{m}(h, \kappa)\right)^{2}:=\frac{h^{4 / p^{\prime}}}{\kappa} f_{I_{m}}\left\|\nabla \mathbf{u}\left(t_{m}\right)-\nabla \mathbf{u}(t)\right\|_{\frac{6 p}{4+p}}^{2} d t+f_{I_{m}}\left\|\mathbf{f}\left(t_{m}\right)-\mathbf{f}(t)\right\|_{2}^{2} d t \\
& +\int_{I_{m}}\left\|\mathbf{F}(\mathbf{D u}(t))-\mathbf{F}\left(\mathbf{D u}\left(t_{m}\right)\right)\right\|_{2}^{2} d t .
\end{aligned}
$$


Let us verify that these quantities fulfil assumption (2.6). First, we observe that, by using the regularity (2.20) on the velocity $\mathbf{u}$, it holds

$$
\kappa \sum_{m=1}^{M} r_{m}^{2}(h, \kappa) \leq c h^{2} \int_{0}^{T}\left\|\nabla^{2} \mathbf{u}(t)\right\|_{\frac{6 p}{p+4}}^{2} d t \leq c h^{2} .
$$

Second, by using the regularity (2.14), the condition (2.15) and the regularity (2.20), Proposition 2.3 (i) and the regularity (2.14), and again the regularity (2.14), we obtain

$$
\begin{aligned}
\kappa \sum_{m=1}^{M} s_{m}^{2}(h, \kappa) \leq & c h^{2} \int_{0}^{T}\|\nabla \mathbf{F}(\mathbf{D u}(t))\|_{2}^{2} d t+h^{2} \frac{h^{4 / p^{\prime}}}{\kappa} \int_{0}^{T}\left\|\nabla^{2} \mathbf{u}(t)\right\|_{\frac{6 p}{4+p}}^{2} d t \\
& +h^{2} \int_{0}^{T}\|\nabla q(t)\|_{p^{\prime}}^{p^{\prime}} d t+h^{2} \int_{0}^{T}\|\mathbf{F}(\mathbf{D u}(t))\|_{2}^{2} d t+h^{2}|\Omega| \delta^{p^{\prime}} \\
\leq & c h^{2} .
\end{aligned}
$$

Third, using Hölder's inequality, several times Lemma 2.6, the regularity (2.20), and the assumption on the regularity of $\mathbf{f}$ we get (since $p \leq 2$ )

$$
\begin{aligned}
\kappa \sum_{m=1}^{M} \rho_{m}^{2} & \leq c \kappa^{2} \int_{0}^{T}\left\|\partial_{t} \nabla \mathbf{u}(t)\right\|_{\frac{\sigma p}{p+4}}^{2} d t+c \kappa^{2} \int_{0}^{T}\left\|\partial_{t} \mathbf{f}(t)\right\|_{2}^{2} d t \\
& \leq c \kappa^{2} .
\end{aligned}
$$

Next, by using Lemma 2.6, the condition (2.15), the regularity (2.20), as well as the regularity (2.20), and the assumption on the regularity of $\mathbf{f}$ we have

$$
\begin{aligned}
\kappa \sum_{m=1}^{M} \sigma_{m}^{2}(h, \kappa) \leq & c \kappa^{2} \frac{h^{4 / p^{\prime}}}{\kappa} \int_{0}^{T}\left\|\partial_{t} \nabla \mathbf{u}(t)\right\|_{\frac{6 p}{4+p}}^{2} d t+c \kappa^{2} \int_{0}^{T}\left\|\partial_{t} \mathbf{f}(t)\right\|_{2}^{2} d t \\
& +c \kappa^{2} \int_{0}^{T}\left\|\partial_{t} \mathbf{F}(\mathbf{D} \mathbf{u}(t))\right\|_{2}^{2} d t \\
\leq & c \kappa^{2} .
\end{aligned}
$$

Finally, since $\mathbf{u}_{h}^{0}=\Pi_{h}^{\text {div }} \mathbf{u}_{0}$, the regularity of $\mathbf{u}_{0}$ and Proposition 2.9 yield

$$
\begin{aligned}
& a_{0}(h)=\left\|\mathbf{u}_{0}-\Pi_{h}^{\text {div }} \mathbf{u}_{0}\right\|_{2} \leq c h\left\|\nabla \mathbf{u}_{0}\right\|_{2} \leq c h, \\
& b_{0}(h)=\left\|\mathbf{D} \mathbf{u}_{0}-\mathbf{D} \Pi_{h}^{\text {div }} \mathbf{u}_{0}\right\|_{p} \leq c h\left\|\nabla^{2} \mathbf{u}_{0}\right\|_{p} \leq c h .
\end{aligned}
$$

Consequently Lemma 2.5 yields for sufficiently small $\kappa$ and $h$ that

$$
\begin{gathered}
\max _{1 \leq m \leq M}\left\|\mathbf{e}_{h}^{m}\right\|_{2}^{2}+\gamma_{1}(1+\Lambda)^{p-2} \kappa \sum_{m=0}^{M}\left\|\mathbf{D} \mathbf{e}_{h}^{m}\right\|_{p}^{2} \leq c\left(h^{2}+\kappa^{2}\right), \\
\max _{1 \leq m \leq M}\left\|\mathbf{D} \mathbf{e}_{h}^{m}\right\|_{p} \leq 1 .
\end{gathered}
$$


Using this, the estimates (3.23)-(3.26) and $\left\|\mathbf{e}_{h}^{m-1}\right\|_{2}^{\theta}\left\|\mathbf{D} \mathbf{e}_{h}^{m-1}\right\|_{p}^{1-\theta}\left\|\mathbf{D} \mathbf{e}_{h}^{m}\right\|_{p} \leq c$ $\left\|\mathbf{D} \mathbf{e}_{h}^{m-1}\right\|_{p}^{2}+c\left\|\mathbf{D} \mathbf{e}_{h}^{m}\right\|_{p}^{2}$, one easily checks that all terms on the right-hand side of (3.22) are bounded, after multiplication by $\kappa$ and summation over $m=1, \ldots, M$, by $c\left(h^{2}+\kappa^{2}\right)$. Thus, we proved

$$
\max _{m=1, \ldots, M}\left\|\mathbf{u}_{h}^{m}-\mathbf{u}\left(t_{m}\right)\right\|_{2}^{2}+\kappa \sum_{m=1}^{M}\left\|\mathbf{F}\left(\mathbf{D} \mathbf{u}_{h}^{m}\right)-\mathbf{F}\left(\mathbf{D u}\left(t_{m}\right)\right)\right\|_{2}^{2} \leq c\left(h^{2}+\kappa^{2}\right),
$$

which is the assertion of Theorem 2.11.

Proof of Corollary 2.13 This corollary is proved in the same way as Theorem 2.11 with the only difference that in all places where (2.19) is used we use (2.21) instead.

Let us illustrate that on the estimate of $I_{2}^{m}(t)$ in (3.8). Using the definition of $b(\cdot, \cdot, \cdot)$ in (1.3), and partial integration we get for all $m=1, \ldots, M$ and $t \in I_{m}$, also using Hölder's inequality with $\left(r, \frac{3 p r}{3 p r-2 p-3}, \frac{3 p}{3-p}\right)$ and $\left(p, \frac{p}{p-1}, \infty\right)$ for $r \in(3,6(p-1))$, respectively, the embeddings $W^{1, p}(\Omega) \hookrightarrow L^{\frac{3 p}{3-p}}(\Omega), \quad W^{1, r}(\Omega) \hookrightarrow L^{\infty}(\Omega)$, Korn's inequality, the embedding $L^{\frac{p}{p-1}}(\Omega) \hookrightarrow L^{\frac{3 p r}{p p r-2 p-3}}(\Omega)$, the interpolation of $L^{\frac{p}{p-1}}(\Omega)$ between $L^{2}(\Omega)$ and $W^{1, p}(\Omega)$, which is possible for $p \in\left(\frac{3}{2}, \frac{8}{5}\right.$, the continuity of $\Pi_{h}^{\text {div }}$ (cf. Proposition 2.9 (ii)), and (2.21)

$$
\begin{aligned}
\left|I_{2}^{m}(t)\right| & \leq \frac{1}{2}\left|\left(\left[\nabla \Pi_{h}^{\operatorname{div}} \mathbf{u}\left(t_{m}\right)\right] \mathbf{e}_{h}^{m-1}, \Pi_{h}^{\operatorname{div}} \mathbf{e}_{h}^{m}\right)\right|+\frac{1}{2}\left|\left(\left[\nabla \Pi_{h}^{\operatorname{div}} \mathbf{e}_{h}^{m}\right] \mathbf{e}_{h}^{m-1}, \Pi_{h}^{\operatorname{div}} \mathbf{u}\left(t_{m}\right)\right)\right| \\
& \leq c\left\|\nabla \Pi_{h}^{\operatorname{div}} \mathbf{u}\left(t_{m}\right)\right\|_{r}\left(\left\|\mathbf{e}_{h}^{m-1}\right\|_{\frac{3 p r}{3 p r-2 p-3}}+\left\|\mathbf{e}_{h}^{m-1}\right\|_{\frac{p}{p-1}}\right)\left\|\mathbf{D} \Pi_{h}^{\operatorname{div}} \mathbf{e}_{h}^{m}\right\|_{p} \\
& \leq c\left\|\mathbf{e}_{h}^{m-1}\right\|_{2}^{\theta}\left\|\mathbf{D} \mathbf{e}_{h}^{m-1}\right\|_{p}^{1-\theta}\left\|\mathbf{D} \mathbf{e}_{h}^{m}\right\|_{p},
\end{aligned}
$$

with $\theta:=\frac{8 p}{5 p-6} \in(0,1]$ for $p \in\left(\frac{3}{2}, \frac{8}{5}\right.$. Using the embedding $W^{1, p}(\Omega) \hookrightarrow L^{2}(\Omega)$ in the last line we also obtain

$$
\left|I_{2}^{m}(t)\right| \leq c\left\|\mathbf{D} \mathbf{e}_{h}^{m-1}\right\|_{p}\left\|\mathbf{D} \mathbf{e}_{h}^{m}\right\|_{p} .
$$

Similar adaptations apply to the treatment of the other terms stemming from the convective term. This proves the assertion.

Due to the presence of the term $\left|\left(\left[\nabla \Pi_{h}^{\text {div }} \mathbf{e}_{h}^{m}\right] \mathbf{e}_{h}^{m-1}, \Pi_{h}^{\text {div }} \mathbf{u}\left(t_{m}\right)\right)\right|$ an extension of the validity of the error estimate for $p \leq \frac{3}{2}$ with the present technique is impossible, even with further regularity assumptions on $\mathbf{u}$.

Acknowledgements The research of Luigi C. Berselli that led to the present paper was partially supported by a grant of the group GNAMPA of INdAM and by the project of the University of Pisa within the grant PRA_2018_52 UNIPI Energy and regularity: New techniques for classical PDE problems.

Funding Open Access funding enabled and organized by Projekt DEAL.

Open Access This article is licensed under a Creative Commons Attribution 4.0 International License, which permits use, sharing, adaptation, distribution and reproduction in any medium or format, as long as you give appropriate credit to the original author(s) and the source, provide a link to the Creative Commons licence, and indicate if changes were made. The images or other third party material in this article are included in the article's Creative Commons licence, unless indicated otherwise in a credit line to the material. If 
material is not included in the article's Creative Commons licence and your intended use is not permitted by statutory regulation or exceeds the permitted use, you will need to obtain permission directly from the copyright holder. To view a copy of this licence, visit http:// creativecommons.org/licenses/by/4.0/.

\section{References}

1. Bartels, S., Růžička, M.: Convergence of fully discrete implicit and semi-implicit approximations of singular parabolic equations. SIAM J. Numer. Anal. 58(1), 811-833 (2020)

2. Belenki, L., Berselli, L.C., Diening, L., Růžička, M.: On the Finite Element approximation of $p$-Stokes systems. SIAM J. Numer. Anal. 50(2), 373-397 (2012)

3. Berselli, L.C., Diening, L., Růžička, M.: Optimal error estimates for a semi implicit Euler scheme for incompressible fluids with shear dependent viscosities. SIAM J. Numer. Anal. 47(3), 2177-2202 (2009)

4. Berselli, L.C., Diening, L., Růžička, M.: Existence of strong solutions for incompressible fluids with shear dependent viscosities. J. Math. Fluid Mech. 12(1), 101-132 (2010)

5. Berselli, L.C., Diening, L., Růžička, M.: Optimal error estimates for semi-implicit space-time discretization for the equations describing incompressible generalized Newtonian fluids. IMA J. Numer. Anal. 25(2), 680-697 (2015)

6. Berselli, L.C., Růžička, M.: Space-time discretization for nonlinear parabolic systems with $p$-structure. IMA J. Numer. Anal. (2020). https://doi.org/10.1093/imanum/draa079

7. Berselli, L.C., Kaltenbach, A., Růžička, M.: Fully discrete, quasi non-conforming approximation of evolution equations. Math. Models Methods Appl. Sci. (2020). arXiv:2007.15449

8. Bothe, D., Prüss, J.: $L^{p}$-theory for a class of Non-Newtonian fluids. SIAM J. Math. Anal. 39(2), 379-421 (2007)

9. Breit, D., Diening, L., Storn, J., Wichmann, J.: The parabolic p-Laplacian with fractional differentiability. IMA J. Numer. Anal. (2020). https://doi.org/10.1093/imanum/draa081

10. Breit, D., Hofmanova, M., Loisel, S.: Space-time approximation of stochastic p-Laplace systems. Tech. Report 1904.03134 (2020)

11. Breit, D., Mensah, P.R.: Space-time approximation of parabolic systems with variable growth. IMA J. Numer. Anal. 40(4), 2505-2552 (2019)

12. Diening, L., Ettwein, F.: Fractional estimates for non-differentiable elliptic systems with general growth. Forum Math. 20(3), 523-556 (2008)

13. Diening, L., Kreuzer, Ch.: Linear convergence of an adaptive finite element method for the $p$-Laplacian equation. SINUM 46, 614-638 (2008)

14. Diening, L., Prohl, A., Růžička, M.: On time-discretizations for generalized Newtonian fluids. In: Birman, M.S., Hildebrandt, S., Solonnikov, V., Uraltseva, N.N. (eds.) Nonlinear Problems in Mathematical Physics and Related Topics II, pp. 89-118. Kluwer/Plenum, New York (2002)

15. Diening, L., Prohl, A., Růžička, M.: Semi-implicit Euler scheme for generalized Newtonian fluids. SIAM J. Numer. Anal. 42, 1172-1190 (2006)

16. Diening, L., Růžička, M.: Strong solutions for generalized Newtonian fluids. J. Math. Fluid Mech. 7, 413-450 (2005)

17. Diening, L., Růžička, M.: Interpolation operators in Orlicz-Sobolev spaces. Numer. Math. 107, 107-129 (2007)

18. Diening, L., Růžička, M., Wolf, J.: Existence of weak solutions for unsteady motions of generalized Newtonian fluids. Ann. Scuola Norm. Sup. Pisa Cl. Sci. V IX, 1-46 (2010)

19. Eckstein, S., Růžička, M.: On the full space-time discretization of the generalized Stokes equations: the Dirichlet case. SIAM J. Numer. Anal. 56(4), 2234-2261 (2018)

20. Prohl, A., Růžička, M.: On fully implicit space-time discretization for motions of incompressible fluids with shear dependent viscosities: the case $p \leq 2$. SIAM J. Numer. Anal. 39, 214-249 (2001)

21. Růžička, M., Diening, L.: Non-Newtonian fluids and function spaces, Nonlinear Analysis, Function Spaces and Applications. In: Proceedings of NAFSA 2006 Prague, vol. 8, pp. 95-144 (2007)

22. Scott, L.R., Zhang, S.: Finite element interpolation of nonsmooth functions satisfying boundary conditions. Math. Comput. 54(190), 483-493 (1990)

23. Süli, E., Tscherpel, T.: Fully discrete finite element approximation of unsteady flows of implicitly constituted incompressible fluids. IMA J. Numer. Anal. 40(2), 801-849 (2019)

24. Tscherpel, T.: Finite element approximation for the unsteady flow of implicitly constituted incompressible fluids. PhD Thesis, University of Oxford (2018) 